\title{
REVUE DES CRUSTACÉS ATYIDAE ET PALAEMONIDAE D'EAU DOUCE DE POLYNÉSIE FRANÇAISE AVEC DESCRIPTION D'UNE NOUVELLE ESPÈCE DE MACROBRACHIUM.
}

\author{
P. KEITH (1), E. VIGNEUX (2)
}

(1) Muséum National d'Histoire Naturelle, 57 rue Cuvier, 75231 PARIS Cedex 05, France.

(2) Conseil Supérieur de la Pêche, le Paraclet, 80440 BOVES, France.

\section{RÉSUMÉ}

Des inventaires de crustacés décapodes ont été réalisés dans les eaux douces de Polynésie française pendant 4 ans. 13 espèces ont été trouvées : deux sont endémiques Caridina rapaensis (Edmonson, 1935) des lles Australes et Macrobrachium feunteuni n. sp. des lles Marquises et qui est décrite ici comme nouvelle. Macrobrachium grandimanus (Randall, 1840) a été capturé pour la première fois dans cette région. Les eaux douces de Polynésie française sont riches et maintenant bien connues.

Mots-clés : Atyoida, Caridina, Macrobrachium, Palaemon, Polynésie française, espèce nouvelle, revue.

\section{A REVIEW OF FRESHWATER ATYIDAE AND PALAEMONIDAE OF FRENCH POLYNESIA WITH DESCRIPTION OF A NEW MACROBRACHIUM SPECIES.}

\section{ABSTRACT}

Inventories of the freshwater crustacean were carried out on French Polynesia rivers over a period of 4 years. 13 species were found: two are endemic, Caridina rapaensis (Edmonson, 1935) from Austral islands and Macrobrachium feunteuni n. sp. from Marquesas islands which is described here as new. Macrobrachium grandimanus (Randall, 1840) was caught for the first time in this area. Freshwaters of French Polynesia are rich and now well known.

Key-words : Atyoida, Caridina, Macrobrachium, Palaemon, French Polynesia, new species, review. 


\section{INTRODUCTION}

La Polynésie française couvre un vaste domaine océanique situé à la limite Est de la province Indo-Pacifique. Elle est formée de 118 îles, comprenant des îles hautes volcaniques et des îles basses coralliennes ou atolls. Ces différentes îles représentent une surface de $3629 \mathrm{~km}^{2}$ de terres émergées, disséminées sur 2,5 millions de $\mathrm{km}^{2}$ d'océan. Elles sont réparties en cinq archipels (Figure 1), dispersés le long d'un axe général sudest, nord-ouest ; il s'agit de :

- l'archipel des Australes (141 $\left.\mathrm{km}^{2}\right)$ : six îles hautes et un atoll,

- l'archipel des Gambier $\left(23 \mathrm{~km}^{2}\right)$ : neuf îles hautes,

- l'archipel des Marquises $\left(997 \mathrm{~km}^{2}\right)$ : onze îles hautes et un atoll,

- l'archipel de la Société (1618 km²) : neuf îles hautes et cinq atolls,

- l'archipel des Tuamotu $\left(850 \mathrm{~km}^{2}\right): 76$ atolls.

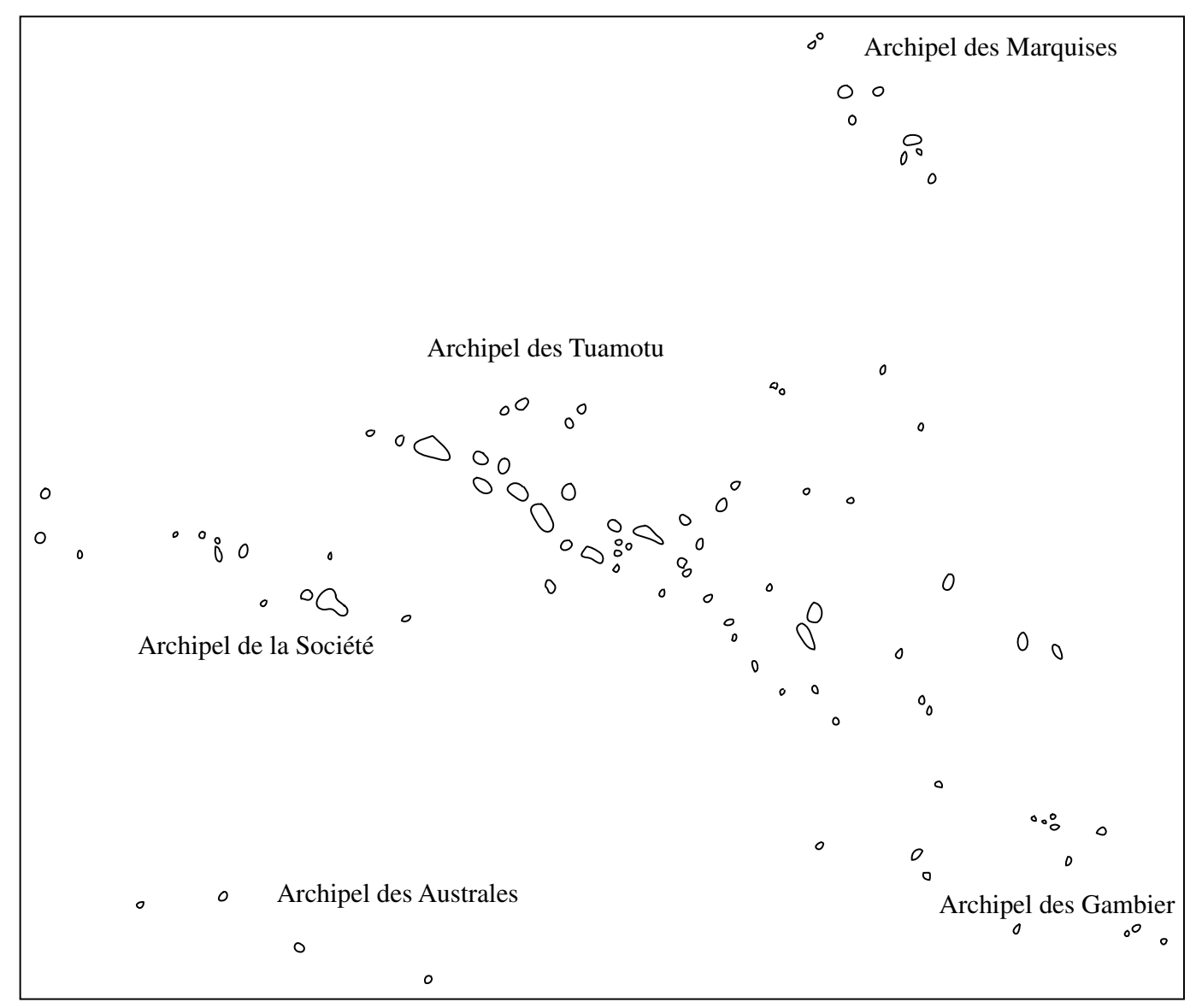

\section{Figure 1}

Carte de la Polynésie française.

\section{Figure 1}

Map of French Polynesia. 
Les publications sur la faune aquatique de ce vaste domaine insulaire sont cependant peu nombreuses et relativement anciennes. De plus, elles ont essentiellement été consacrées aux poissons marins. La faune de l'archipel de la Société a été la plus étudiée notamment par KENDALL et GOLDSBOROUGH (1911), FOWLER (1932), HERRE (1931, 1932), POLL (1942), EGE (1939), PLESSIS (1973) et RANDALL (1973a, b). En raison de leur isolement géographique, les autres archipels ont été beaucoup moins prospectés. Toutefois, la faune de l'archipel des Marquises a été étudiée par FOWLER (1932), PLESSIS et MAUGE (1978) et RANDALL (1985). L'archipel des Gambier a été prospecté par SEURAT (1934) et FOURMANOIR et al. (1974). Trois îles de l'Archipel des Australes ont été prospectées, Tubuaï et Rurutu (PLESSIS, 1980 ; MARQUET et GALZIN, 1992) et celle de Rapa (EGE, 1939 ; RANDALL, 1978 ; PLESSIS, 1987 ; RANDALL et al., 1990).

Les publications spécifiques aux crustacés d'eau douce de Polynésie française sont rares dans la littérature. SEURAT (1934) signalait 5 espèces à Tahiti et 3 espèces à Mangareva (Gambier), ADAMSON (1935) en signalait 6 aux Marquises et EDMONSON (1935) décrit une nouvelle caridine des îles Rapa. Des travaux généraux de révision ou de biogéographie feront référence aux espèces présentes en Polynésie (BOUVIER, 1925 ; EDMONSON, 1951 ; HOLTHUIS, 1950, 1952, 1980 ; HOBBS et HART, 1982 ; CHACE, 1983) mais il faut attendre MARQUET (1991) pour qu'une première synthèse des connaissances soit disponible.

Malgré l'énorme travail réalisé par ce dernier auteur, plusieurs îles de Polynésie française avec un réseau hydrographique important n'avaient pu être prospectées. Les connaissances sur les espèces de crustacés d'eau douce méritaient ainsi d'être augmentées et d'être mises à jour afin d'améliorer les connaissances pour la gestion des espèces et des populations.

Une série d'inventaires a donc été réalisée entre 1997 et 2000 sur l'essentiel des rivières pérennes à l'initiative de la Délégation à l'Environnement, de l'EPHE (CRIOBE), du Muséum National d'Histoire Naturelle (MNHN) et du Service du développement rural (SDR).

\section{MATÉRIEL ET MÉTHODES}

Les sondages ont été réalisés dans tous les types de milieux des cinq archipels constituant la Polynésie française : les estuaires au taux de salinité variable ; les plans d'eau, lacs ou étangs ; les rivières au cours rapide alternant avec des zones plus lentes.

Trois méthodes d'inventaire ont été utilisées : la pêche électrique, la pêche à la senne et la pêche à l'épuisette. Pour la première un générateur de courant de type Martinpêcheur a été utilisé. Le Martin-pêcheur (GOSSET et al., 1971) est un appareil portable à batterie, d'une puissance utile de $180 \mathrm{~W}$, pesant au total $12 \mathrm{~kg}$. II fournit des impulsions rectangulaires à fréquence fixe, $100 \mathrm{~Hz}$ ou $400 \mathrm{~Hz}$, à rapport cyclique réglable de 5 à $25 \%$. Il comprend trois tensions de sortie : 150, 200 et 300 V. Cet appareil a été conçu pour pêcher dans des eaux dont la conductivité est comprise entre 50 et $1000 \mu \mathrm{S} . \mathrm{cm}^{-1}$. La pêche a été pratiquée à pied, en remontant la rivière de façon à ce que l'eau reste claire devant le pêcheur et de manière à ce que les animaux tétanisés par le courant électrique soient entraînés vers l'épuisette par le courant d'eau.

Pour la pêche à la senne, effectuée en estuaire exclusivement, le principe repose sur l'utilisation d'un filet avec une poche dont l'une des extrémités est un point fixe sur la berge et l'autre est tirée en demi cercle dans l'eau jusqu'à être ramené sur la berge vers le point fixe. 
La pêche à l'épuisette est utilisée en eau peu profonde et claire pour les espèces peu sensibles à l'électricité. Celles-ci sont repérées à vue à distance ou dans l'eau en apnée et poussées vers les épuisettes.

Les espèces capturées sont immédiatement photographiées in vivo sur le terrain afin d'éviter les pertes de coloration puis les espèces sont déterminées et conservées ou relâchées suivant les cas. Les échantillons conservés sont fixés dans le formol (10\%) puis stockés dans l'alcool $(70 \%)$. Une collection de référence a été constituée au MNHN de Paris.

\section{RÉSULTATS}

13 espèces de crustacés décapodes ont été recensées en Polynésie française (4 Atyidae et 9 Palaemonidae). Le Tableau I indique leur répartition dans les 5 archipels. Dans le texte qui suit, après le nom scientifique de chaque espèce figurent les noms vernaculaires polynésiens lorsqu'ils sont connus ; un $M$ signifie qu'il s'agit du nom marquisien, un $\mathrm{S}$ du nom tahitien, un $\mathrm{F}$ du nom français.

\section{Tableau I}

Liste des espèces d'Atyidae et de Palaemonidae présentes en Polynésie française.

\section{Table I}

List of Atyidae and Palaemonidae species of French Polynesia.

\begin{tabular}{|l|c|c|c|c|c|}
\hline \multicolumn{1}{|c|}{ ESPÈCES } & Société & Marquises & Australes & Gambier & Tuamotu \\
\hline Atyidae & & & & & \\
Atyoida pilipes & + & + & + & + & - \\
Caridina rapaensis & - & - & + & - & - \\
Caridina serratirostris & + & - & + & - & - \\
Caridina weberi & + & + & + & - & - \\
Palaemonidae & & & & & \\
Macrobrachium aemulum & + & - & + & + & - \\
Macrobrachium australe & + & + & + & + & - \\
Macrobrachium lar & + & + & + & + & - \\
Macrobrachium latimanus & + & + & + & + & - \\
Macrobrachium lepidactyloides & + & - & - & - & - \\
Macrobrachium grandimanus & + & - & - & - & - \\
Macrobrachium feunteuni $\mathrm{n} . \mathrm{sp}$. & - & + & - & - & - \\
Palaemon concinnus & + & - & + & - & - \\
Palaemon debilis & - & - & + & + & + \\
& & & & & $\mathbf{6}$ \\
\multicolumn{2}{|c|}{ Total } & $\mathbf{1 0}$ & $\mathbf{6}$ & $\mathbf{1 0}$ & + \\
\hline
\end{tabular}




\section{Famille des Atyidae}

Atyoida pilipes (Newport, 1847) ; nipuu, hinako (M : marquisien), crevette, saltarelle koros ( $F$ : français) (Photos 1 et 2).

Le genre se distingue par des yeux bien développés, un rostre sans épines dorsales, par l'absence d'épines supra-orbitales mais la présence d'épines antennaires sur le bord antérieur de la carapace (CHACE, 1983). Les péréiopodes sont dépourvus d'exopodites, mais les premières et deuxièmes paires possèdent des chélipèdes avec des touffes de soies, permettant de filtrer l'eau. L'espèce se différencie grâce à son rostre un peu incurvé vers le bas, non armé ou portant simplement une dent ventralement, moins fréquemment deux et très rarement trois. On trouve des dents réduites sur le bord postérieur du telson. On peut observer 5 paires d'épipodites à la base des pattes antérieures (HOLTHUIS, 1950). Le dimorphisme sexuel se caractérise notamment par l'absence d'éperons sur le mérus de la troisième paire de pattes chez les grands mâles (HOBBS et HART, 1982). Les femelles sont plus grandes que les mâles. C'est une espèce protandre. La taille moyenne est de 35 à $40 \mathrm{~mm}$.

Les adultes vivent totalement en eau douce mais il semble que les jeunes éclosent en eau saumâtre ou en mer où ils se développent quelques semaines avant de retourner en rivière. Les adultes ont une préférence pour les eaux fraîches et très bien oxygénées (FOSSATI et al., 2002) dans les cours moyens et supérieurs. Ils sont en général dans les zones où le courant est le plus fort (petites cascades...) sous la lame d'eau, accrochés sur les rochers. Leur régime alimentaire est de type microphage. Elles filtrent l'eau à l'aide des touffes de soies situés à l'extrémité des chélipèdes. C'est une espèce très largement répartie dans la zone Indo-Pacifique.

Caridina rapaensis (Edmondson, 1935) (Photos 3 et 4)

Les yeux sont bien développés mais courts. Le rostre est mince, légèrement incurvé vers le bas, s'allongeant jusqu'à la moitié du second segment du pédoncule antennulaire. Les dents du rostre sont petites et peu nombreuses, plus fortes sur le bord supérieur que sur le bord inférieur (EDMONDSON, 1935). En général, celles du haut occupent le quart distal. Elles sont au nombre de zéro à quatre sur chaque. L'épine sub-orbitale possède une large base. Le premier segment des antennules est légèrement concave sur sa partie dorsale. Le second segment est plus court que le premier et le troisième segment est aussi long que le second. Les bords ventro-latéraux du second et du troisième segment sont munis d'une dense frange de longues soies plumeuses. La première paire de péréiopodes a un propode plus long que le carpe et le dactyle est aussi long que le bord supérieur de la paume. La seconde paire est plus mince que la première et possède un propode aussi long que le carpe. Le telson possède six paires de petites épines sur la face supérieure et une petite dent médiane sur la face inférieure. Son extrémité postérieure présente 9 soies plumeuses ainsi qu'un long et fin aiguillon à chaque angle (EDMONDSON, 1935). La taille varie de 8 à $14 \mathrm{~mm}$.

Son régime alimentaire est plutôt de types microphage et phytophage. Elle filtre les particules organiques de l'eau à l'aide de ses appendices buccaux. On la trouve du cours inférieur au cours supérieur des rivières. Cette espèce est endémique de l'archipel des Australes.

\section{Caridina serratirostris (De Man, 1892) (Photos 5 et 6)}

Les yeux sont bien développés. Le rostre rectiligne est légèrement incurvé vers le haut. II porte 19 à 26 dents sur sa face dorsale dont 6 à 8 sont placées derrière l'orbite oculaire et 6 à 8 dents sur sa face ventrale. L'épine antennaire est visible et placée sous l'angle orbitaire inférieur. Le second segment du pédoncule antennulaire est très long et 
fin. Le carpe du premier péréiopode est plus long que le mérus, 3,5 fois plus long que large et aussi long que le propode. Chez le deuxième péréiopode, le carpe est 10 fois plus long que large et bien plus long que le propode. Le telson porte 4 à 5 paires d'épines sur la face dorsale. Le stylocérite est très long et dépasse la base du pédoncule antennulaire. La coloration du corps est variable : de hyalin à rose orange ou carmin. Taille : 10 à $20 \mathrm{~mm}$.

Cette espèce vit dans les eaux saumâtres des estuaires et dans les eaux stagnantes peu profondes des étangs côtiers. Son régime alimentaire est de type détritivore. Elle se nourrit de détritus, d'algues sur les rochers, qui sont raclés et ramassés grâce à ses deux pinces terminées par de longues soies.

Elle pond de petits œufs $(0,35$ à $0,5 \mathrm{~mm}$ ) (KEITH et al., 1999). Cette espèce fréquente la zone Indo-Pacifique de Madagascar, des Seychelles, Maurice et La Réunion à la Polynésie française et la Nouvelle-Calédonie.

Caridina weberi (De Man, 1892) ; nipuu, hinako (M) ; saltarelle nez-camus (F) (Photos 7 et 8 )

Les yeux sont bien développés. L'espèce est très caractéristique par ses extensions infra-orbitaires formant un étroit liseré au-dessus de l'épine antéro-latérale, elle-même fort distincte. Son prolongement préanal est caréné et saillant en arrière (FENNER et CHACE, 1983). L'armature des doigts des péréiopodes 3 et 4 est spécifique car la première épine est beaucoup plus forte que les suivantes. Le rostre peut être infléchi dans sa moitié antérieure (HOLTHUIS, 1950). II est composé de douze dents sur la partie supérieure et de quatre sur la partie inférieure. Le propode de la première paire de péréiopodes est légèrement plus grand que le carpe tandis que pour la seconde paire, c'est le carpe qui est plus long que le propode. La partie postérieure du telson porte dix longues soies. Sa coloration est souvent hyaline à blanc rosé avec quelques chromatophores plus foncés disséminés sur les flancs. La taille moyenne des différentes variétés de cette espèce est de $22 \mathrm{~mm}$, mais elle peut atteindre plus de $30 \mathrm{~mm}$.

Cette espèce a un régime de type détritivore. Elle se nourrit de détritus, d'algues sur les rochers, qui sont raclés et ramassés grâce à ses deux pinces terminées par de longues soies.

\section{Famille des Palaemonidae}

\section{Palaemon concinnus (Dana, 1852) (Photos 9 et 10)}

Les yeux sont bien développés. Le rostre est légèrement incurvé vers le haut. Sa face dorsale comporte de 5 à 8 dents, sa face ventrale entre 3 à 7 dents. La première dent de la rangée dorsale se trouve derrière la limite de l'orbite oculaire (HOLTHUIS, 1950). Les dents placées ventralement sont dans les deux tiers antérieurs du rostre. L'extrémité de la face dorsale du rostre est lisse excepté pour une petite dent sub-apicale. Le céphalothorax est pourvue d'une épine branchiostège forte, située sur sa marge antérieure. Les deuxième et troisième segments antennulaires sont plus courts et plus petits que le premier. Le flagelle antennulaire supérieur a ses deux ramifications fusionnées sur 6 à 10 articles et la partie libre du rameau le plus court est composé de 21 à 30 articles. Les doigts des premiers péréiopodes sont minces et d'une longueur égale ou supérieure à celle de la paume. Le carpe est allongé d'environ 2,5 à 3 fois la longueur de la pince. Les seconds péréiopodes sont également minces et élancés, mais plus forts que la première paire. Le telson est allongé et triangulaire. Taille : de 60 à $65 \mathrm{~mm}$.

Cette crevette fréquente les eaux saumâtres des estuaires ou des étangs côtiers. Palaemon concinnus fréquente toute la zone Indo-Pacifique comprise entre les côtes Est de l'Afrique, la Nouvelle-Calédonie et la Polynésie française. 


\section{Palaemon debilis (Dana, 1852) (Photos 11 et 12)}

L'espèce est caractérisée par un rostre très long et fortement incurvé vers le haut. Au niveau dorsal, les dents du rostre sont séparées par de grands espaces, sauf pour la seconde et la troisième dent postérieure qui sont proches l'une de l'autre. L'extrémité de la face dorsale du rostre est lisse exceptée pour une petite dent sub-apicale. Sur la face inférieure, les dents ont une double rangée de soies. Le bord supérieur du rostre porte dans sa moitié postérieure de 2 à 8 dents, généralement 5 , dont la première est placée derrière la limite de l'orbite oculaire et la seconde légèrement en avant (HOLTHUIS, 1950). Le bord inférieur porte de 3 à 10 dents, généralement 6 , réparties dans les $3 / 4$ antérieurs. Le flagelle antennulaire supérieur a ses deux ramifications fusionnées sur 7 à 14 articles ; la partie libre du rameau le plus court est d'une taille équivalente à celle de la partie soudée. Le céphalothorax est pourvu d'une épine branchiostège plus courte que l'épine antennaire. La première paire de péréiopodes atteint et peut même dépasser le bout du scaphocérite. Les doigts égalent ou excèdent la longueur de la paume (HOLTHUIS, 1950). Le carpe est élancé, d'une longueur équivalente ou double de celle de la pince. Le mérus est bien plus court que le carpe. La deuxième paire de péréiopodes est plus grande et les pinces dépassent le scaphocérite. Les doigts ne sont pas armés et se ferment sur toute leur longueur ; ils mesurent les $2 / 3$ de la paume. Le telson a deux paires d'épines dorsales placées dans le milieu et au $3 / 4$ de la longueur. $P$. debilis est presque transparente et mesure jusqu'à $25 \mathrm{~mm}$.

Cette espèce est rencontrée principalement le long des côtes, en eau saumâtre dans les estuaires et parfois dans la partie basse du cours inférieur des cours d'eau. Son régime alimentaire est de type opportuniste. Elle se nourrit notamment de résidus de matière organique (détritus, algues...). L'espèce a une répartition Indo-Pacifique, elle est présente des îles Hawaï à la Polynésie française (Iles Australes, Tuamotu, Gambier) et à la Nouvelle-Calédonie.

Macrobrachium aemulum (Nobili, 1906) ; bouquet de Nouméa (F) (Photos 13 et 14)

Le rostre est court, sans crête basale distincte et élevée. La face supérieure du rostre comporte 4 à 6 dents placées derrière l'orbite oculaire (HOLTHUIS, 1950, 1980). La seconde paire de péréiopode possède un carpe plus long que le mérus. Les yeux sont bien développés, avec une cornée globuleuse plus large que le pédoncule et pigmentée. Le pédoncule antennulaire est composé de trois articles dont le premier est plus large et plus grand que les deux suivants. L'antennule se compose de deux flagelles. Le telson est allongé, triangulaire et pourvu de deux paires d'épines sur la partie supérieure, dont la plus antérieure se situe au milieu du telson et la deuxième entre cette paire antérieure et le bord postérieur. Le dimorphisme sexuel est visible grâce à la pince proéminente, très longue et fine du mâle adulte. Les doigts ont, au plus, une ou deux dents sur la partie proximale du tranchant du bord interne, tandis que le reste est assez uniforme. Les juvéniles sont transparents, lisses et légèrement tachetés de brun-rouge. Leur carapace est beaucoup moins épaisse que celle des adultes ; leur rostre est relativement plus long et élancé et le sixième segment abdominal est beaucoup plus allongé. Le telson se termine toujours par une pointe effilée. Chez les adultes, les mâles sont en principe plus grands que les femelles. La coloration est brun-rose avec des zébrures caractéristiques sur le céphalothorax. La taille de cette espèce varie de 60 à 85 mm (KEITH et al., 1999).

Le biotope de $M$. aemulum est situé strictement en eau douce dans la partie inférieure à moyenne des cours d'eau. Après l'éclosion, les larves passent par de nombreux stades planctoniques. Le développement de stades post-larvaires se fait en milieu marin à saumâtre. Le régime alimentaire de l'espèce est varié et plutôt de type omnivore (KEITH et al., 1999). On rencontre M. aemulum dans la zone Indo-Ouest Pacifique, plus précisément entre les côtes du Sud-Est de I'Inde jusqu'en NouvelleCalédonie et en Polynésie française. 
Macrobrachium australe (Guérin et Méneville, 1838) ; 'oura 'itara (S : tahitien) ; kuà (M) ; chevrette (F) (Photos 15 et 16)

Cette espèce bras se caractérise par un rostre dont l'extrémité est dirigée vers le haut. La face dorsale du rostre possède de 9 à 13 dents, dont 2 ou 3 derrière l'orbite oculaire. La partie ventrale du rostre possède de 2 à 6 dents. Présence d'une épine hépatique sur le céphalothorax ; absence de l'épine branchiostège. Les pinces longues et effilées sont nettement inégales chez le mâle adulte. Le carpe, plus long que le mérus, est aussi long que la pince (HOLTHUIS, 1950, 1980). La femelle, moins grande, a ses deux pinces de la même longueur mais plus petites que celles des mâles. Les doigts sont minces. De couleur brun clair, le corps est plutôt transparent avec, sur les côtés du céphalothorax, trois rayures brun rouge à carmin et sur tout le corps des points rouge brun à noir. Les rayures sur le céphalothorax, bien visibles chez les jeunes spécimens et les femelles, sont parfois estompées chez les mâles adultes. Taille : de 7 à $8 \mathrm{~cm}$ pour la femelle et 10 à $12 \mathrm{~cm}$ pour le mâle (KEITH et al., 1999).

L'espèce vit dans les cours inférieur et moyen des rivières où elle se réfugie dans les cavités rocheuses ou dans les vasques. Son habitat varie tout au long du cycle biologique. On la trouve aussi en zone d'estuaire sous les berges et dans les herbiers. La reproduction s'effectue en eau douce ou saumâtre. La période d'incubation est d'environ 18 jours. Comme pour les autres Macrobrachium, les larves sont phototropes et planctoniques, elles ne survivent pas plus de 4 à 5 jours dans l'eau douce et migrent rapidement en eau saumâtre ou marine (GRAND, 1972). Les juvéniles mesurant de 25 à $30 \mathrm{~mm}$ effectuent des migrations de la mer vers l'intérieur des terres (KEITH et al., 1999). Ils arrivent à franchir des cascades de plusieurs dizaines de mètres de haut en rampant le long des parois rocheuses humides. Ils se nourrissent de petits vers, de petits crustacés, d'algues et de détritus organiques. Plutôt active la nuit, cette espèce passe la journée à l'abri sous les pierres. Elle a un régime alimentaire de type omnivore, plutôt opportuniste. Cette espèce a une très large distribution dans les océans Indien et Pacifique. II se rencontre à Madagascar, à La Réunion, dans l'archipel Malais et à travers la Polynésie, jusqu'aux îles Marquises.

Macrobrachium grandimanus (Randall, 1840) ; bouquet Hawaïen (F) (Photos 17

L'espèce possède un rostre rectiligne et légèrement dirigé vers le haut. Celui-ci possède 14 dents sur la face supérieure, dont 4 à 5 en arrière de l'orbite oculaire, et 4 sur la face inférieure. La paume chez le mâle est plus grande que le carpe ( 1,1 fois) et 1,5 fois plus grande que le mérus. Le propode est 2,5 fois plus grand que le carpe et 3 fois plus grand que le mérus. Le doigt est presque aussi long que la paume (HOLTHUIS, 1950, 1980). Le corps est hyalin mais parcouru de petites taches rouges nombreuses et disséminées sur tout le corps. Certaines taches regroupées forment des lignes. On peut ainsi distinguer une ligne médiane sur le rostre, une ligne discontinue de chaque côté de l'abdomen et 4 à 5 taches en forme de virgule sur le céphalothorax. Les pattes possèdent une alternance d'anneaux rouges et hyalins. On distingue parfois chez les femelles et chez les juvéniles des anneaux oranges fluorescents à la base des pinces. Taille : l'espèce peut atteindre $7 \mathrm{~cm}$ (KEITH et al., 1999).

Macrobrachium grandimanus préfère la partie basse du cours inférieur des rivières et les estuaires. Elle dépasse rarement la limite supérieure de la zone soumise à l'influence de la marée et s'aventure peu dans la zone dulçaquicole stricte. Elle préfère les eaux lentes où elle s'abrite parmi la végétation immergée des berges. Elle semble avoir un régime alimentaire de type omnivore (détritus organiques, végétaux...).

Cette espèce est connue dans la région Pacifique à Hawaï, Fidji, Tonga, les îles Ryukyu, Hainan et la Nouvelle-Calédonie. Elle a été trouvée pour la première fois en Polynésie, à Moorea en 1997 dans le cadre de la réalisation de ce travail. 
Macrobrachium latimanus (Von Martens, 1868) ; 'oura 'onana, 'ava féefée (S) ; pimata, vaeaei (M) ; bouquet, chevrette montagne (F) (Photos 19 et 20)

L'espèce se distingue par son rostre court et trapu, au bord supérieur dirigé vers le haut et dont la longueur correspond à peu près à la moitié du céphalothorax chez les mâles et un peu plus chez les femelles. Le rostre possède entre 5 et 7 dents sur la face supérieure et entre 1 et 3 dents sur la face inférieure (HOLTHUIS, 1950, 1980). Les yeux sont bien développés, avec une cornée globuleuse plus large que le pédoncule. Le pédoncule antennulaire est composé de trois articles dont le premier est plus large et plus grand que les deux suivants. Le telson est allongé, triangulaire et pourvu de deux paires d'épines sur la partie supérieure, dont la plus antérieure se situe au milieu du telson et la deuxième entre cette paire antérieure et le bord postérieur.

Chez le mâle, la pince du plus grand chélipède est massive et presque aussi longue que le carpe, le mérus et l'ischion réunis (latimanus = large main). Les doigts sont armés sur leur bord interne d'une dizaine de fortes dents et ils ont une taille sensiblement équivalente. Le mérus est cylindrique, plus petit et moins gros que les doigts. Le carpe est court et globuleux. L'ischion est aussi cylindrique et mesure environ la moitié du mérus. Les trois derniers péréiopodes possèdent, excepté sur leur dactyle, des rangées longitudinales de spinules. Ces spinules sont absentes chez les jeunes. Les pattes de la deuxième paire de chélipèdes des femelles sont identiques en forme et en taille et les doigts possèdent 11 à 14 dents rapprochées (HOLTHUIS, 1950, 1980). La coloration est brun-rose à orange avec souvent des points bleus le long de l'abdomen. Les seconds chélipèdes, en particulier chez le mâle, sont brun-orange avec l'extrémité noire. La taille maximum rencontrée avoisine les $130 \mathrm{~mm}$.

Cette espèce fréquente surtout les parties moyenne et supérieure des rivières dans les secteurs à courant moyen à fort et les vasques des cascades. Elle préfère les fonds caillouteux avec des blocs et des rochers qui offrent des caches importantes. La température de l'eau dans ces zones ne dépasse que très rarement $20^{\circ} \mathrm{C}$ et l'oxygénation est bonne. Son aire de répartition s'étend sur toute la façade Ouest du Pacifique, allant du Japon à la Polynésie et aux Fidji, jusqu'aux côtes Ouest de l'Inde.

Macrobrachium lar (Fabricius, 1798) ; 'oura 'pape, 'oura 'oiha'a (S) ; kuà (M) ; chevrette, bouquet singe (F) (Photos 21 et 22)

Son rostre fusiforme et légèrement concave dans sa partie dorsale, atteint ou dépasse le pédoncule antennulaire. Sa face dorsale est munie de 7 ou 10 dents (dont 2 ou 3 dents en arrière de l'orbite oculaire). La face ventrale du rostre porte 3 ou 4 dents, mais généralement 3. Présence d'une épine hépatique sur le céphalothorax ; absence de l'épine branchiostège. La première paire de péréiopodes est mince et élancée, ses doigts sont aussi longs que sa paume. Le carpe est un peu moins long que le mérus. La couleur du corps varie du bleu clair ou bleu turquoise au brun voire rouge. Les femelles ovigères ont souvent une teinte bleu sombre. La chevrette est l'espèce de crustacé d'eau douce indigène la plus grosse de la Polynésie française. Le mâle est plus grand et plus élancé que la femelle qui est plus trapue. Le corps peut parfois dépasser $20 \mathrm{~cm}(30-35 \mathrm{~cm}$ avec les pinces) (HOLTHUIS, 1950, 1980).

Le choix de l'habitat varie selon le cycle biologique de l'espèce. Celle-ci colonise les cavités rocheuses dans les cours d'eau oxygénés, les vasques recevant des cascades, les herbiers, mais aussi la proximité des embouchures. La reproduction s'effectue en eau douce ou saumâtre. Les œufs sont relativement petits et plus de 40000 peuvent être portés par une femelle. La période d'incubation est d'environ 20 jours (ATKINSON, 1977). Les larves passent par de nombreux stades planctoniques de type zoé en milieu saumâtre ou marin. La copulation intervient à l'issue d'une parade nuptiale. Les juvéniles effectuent leur migration vers les eaux douces lorsqu'ils atteignent une taille de 30 à $35 \mathrm{~mm}$. Ils 
remontent le courant et franchissent les chutes en rampant sur les substrats humides (KEITH et al., 1999). L'alimentation de cette espèce est variée et plutôt de type omnivore.

Macrobrachium lar possède une très large répartition dans la zone Indo-Pacifique des côtes Est-Africaines, aux Seychelles, à Madagascar, à l'île Maurice et jusqu'aux îles Marquises en Polynésie française où elle est parfois de teinte orangée.

\author{
Macrobrachium lepidactyloides (De Man, 1892) ; bouquet Malais (F) (Photos 23 \\ et 24)
}

Le rostre est presque droit, légèrement convexe au-dessus des yeux. II atteint en général l'extrémité du second segment du pédoncule antennulaire, parfois le milieu du $3^{\text {ème }}$ segment. Le bord supérieur porte 10 à 13 dents. 5 à 7 (6 en général) sont placées derrière la limite postérieure de l'orbite. Ces dents sont réparties régulièrement le long du rostre. La première dent est placée à peu près au milieu du céphalothorax. Le bord inférieur porte 2 à 3 dents. Les seconds péréiopodes des grands mâles sont très inégaux. Les doigts sont généralement plus fins et plus longs que la paume. La partie proximale des doigts possède de grandes dents : le dactyle porte 2 à 3 dents. Entre la plus distale des grandes dents et la pointe du dactyle, une double rangée d'une douzaine de dents aiguës est présente. La paume est généralement 2 fois moins longue que haute, elle est couverte de nombreux spinules jusque sur les doigts. Le carpe est légèrement plus long que le mérus chez les adultes ; il est parfois plus court chez les jeunes. Dans sa partie postérieure, le mérus devient plus étroit, presque la moitié moins large qu'antérieurement. Le mérus n'atteint pas la moitié de la longueur de l'ischion. Chez la femelle ou le jeune mâle, les 2 pinces sont de taille et de forme voisines. La paume de la plus petite patte marcheuse est plus courte que les doigts ; les doigts de cette patte ont de longues soies sur leur surface extérieure, tandis que le bord coupant porte 2 à 4 très petites dents dans la partie proximale. L'espèce est orangée à brune. Taille : 3 à $7 \mathrm{~cm}$.

Macrobrachium lepidactyloides fréquente plutôt la partie haute du cours inférieur des rivières. Elle préfère les zones courantes de type radier avec des contre-courants. Elle semble avoir un régime alimentaire de type opportuniste : elle se nourrit de débris organiques, d'algues. Les œufs font un diamètre de 0,45 à $0,6 \mathrm{~mm}$; ils sont nombreux et petits (HOLTHUIS, 1950).

Cette espèce a une large répartition Indo-Pacifique, de l'archipel Malais (Philippines, Indonésie), aux Fidji, aux îles Lesser Sunda et à la Polynésie française. En Polynésie, l'espèce type a été trouvée à Raiatea.

Macrobrachium feunteuni, novem species ; kuà (M) (Photos 25 à 33)

\title{
Matériel examiné
}

Holotypes : MNHN-Na13767, 1 mâle $(32,3 \mathrm{~mm})$, Nuku Hiva (îles Marquises, Polynésie Française), rivière Hakaui, 10 février 2000, mission Julien1 coll. (Keith, Vigneux, Feunteun, Cressens).

Paratypes: MNHN-Na13768, 1 mâle $(34,3 \mathrm{~mm})$ et 1 femelle $(27,7 \mathrm{~mm})$, Nuku Hiva (îles Marquises, Polynésie Française), rivière Hakaui, 10 février 2000, mission Julien1 coll. (Keith, Vigneux, Feunteun, Cressens) ; MNHN-Na13769, 1 mâle $(25,2 \mathrm{~mm})$ et 4 femelles (31,5 mm ; 31,1 mm ; $32 \mathrm{~mm} ; 35,8 \mathrm{~mm}$ ), Hiva Oa (îles Marquises, Polynésie Française), rivière Vaioa, 13 février 2000, mission Julien1 coll. (Keith, Vigneux, Feunteun, Cressens). 


\section{Diagnose}

Macrobrachium feunteuni n. sp. est caractérisé par 9 à 11 dents sur le bord supérieur du rostre dont 6 à 7 placées derrière la limite de l'orbite, par une distance entre la première dent et la limite postérieure de l'orbite représentant 24,6 à $30,7 \%$ de la longueur du céphalothorax, par une longueur de la carapace portant les dents rostrales représentant 51 à $56,2 \%$ de la longueur du céphalothorax, par des doigts de la plus grande patte de la deuxième paire de péréiopodes des mâles en général plus courts que la paume et par un carpe de la deuxième paire de péréiopodes des mâles toujours plus long que le mérus.

\section{Description générale}

Les caractéristiques morphométriques sont présentées au Tableau II.

Le rostre est presque droit, légèrement convexe au-dessus des yeux, il est étroit et presque 3 fois plus long que large. II atteint en général l'extrémité du second segment du pédoncule antennulaire, parfois le milieu du $3^{\text {eme }}$ segment mais ne dépasse jamais ce pédoncule. Le bord supérieur porte 9 à 11 dents, 6 à 7 (6 en général) sont placées derrière la limite postérieure de l'orbite. Ces dents sont réparties régulièrement le long du rostre. Elles sont courtes et petites. La première dent est placée à peu près au milieu du céphalothorax, la distance entre cette dent et la limite postérieure de l'orbite représente 24,6 à $30,7 \%$ de la longueur totale du céphalothorax. Le bord inférieur porte 1 à 2 dents. L'épine hépatique est plus petite que l'antennaire et est placée en arrière et au dessus d'elle et non sur la même ligne.

Le telson est 1,3 à 1,6 fois aussi long que le $6^{\text {ème }}$ segment abdominal. Des 2 paires d'épines dorsales, la paire antérieure est située au niveau ou juste derrière le milieu du telson, la paire postérieure à peu près à la moitié de la distance entre la paire antérieure et la limite postérieure du telson.

Les seconds péréiopodes des grands mâles sont très inégaux. Le plus grand péréiopode chez un adulte est plus grand que tout le corps. Les doigts sont pratiquement de même taille que la paume. La partie proximale des doigts possède de grandes dents : le dactyle porte 4 à 5 dents dans le tiers proximal et une dent dans le dernier tiers distal. Entre la plus distale des grandes dents et la pointe du dactyle, une double rangée de 6 à 10 dents est présente. Le doigt fixe possède 2 grandes dents, l'une à la base du doigt, l'autre à peu près au tiers de sa longueur et 5 dents plus petites et jointives avec la grosse dent de la base du doigt. Une double rangée de dents est présente entre la grande dent distale et le doigt fixe. La paume est généralement plus compressée. La paume et les doigts ont de nombreuses spinules. Le carpe est légèrement plus long que le mérus chez les adultes ; il est parfois plus court chez les jeunes. L'ischion représente 52 à $59 \%$ du mérus. Tous les joints sont recouverts de nombreuses spinules.

Le chélipède (P2) chez le mâle adulte dépasse de la pince et du carpe le scaphocérite.

Chez la femelle ou le jeune mâle, les chélipèdes (P2) sont de taille et de forme voisines. La paume de la plus petite pince est légèrement plus courte que les doigts. De longues soies sont visibles sur les doigts. Le doigt mobile possède une dent au milieu de sa longueur et 4 dans sa partie proximale. Le doigt fixe possède une dent au milieu de sa longueur et 5 courtes en partie proximale.

La troisième patte des mâles a un propode qui est plus de 2 fois plus long que le dactyle. Le carpe mesure les $2 / 3$ de la longueur du propode. Le mérus est plus long que le propode. L'ischion fait à peu près la moitié du mérus. 
Tableau II

Caractéristiques morphométriques de Macrobrachium feunteuni n. sp.

Table II

Morphometric characteristics of Macrobrachium feunteuni n. sp.

\begin{tabular}{|c|c|c|c|c|c|c|c|c|c|c|c|}
\hline & Taille $(\mathrm{mm})$ & Dents S & Dents I & LC & D1dent & $\%$ LC/D1dent & LCD & $\%$ LCD/LC & 6SA & Telson (T) & $\%$ 6SA/T \\
\hline \multicolumn{12}{|l|}{ MNHN-Na13767 } \\
\hline Mâle 1 (Holotype) & 32,3 & 9,0 & 2,0 & 11,9 & 3,7 & 30,7 & 6,3 & 52,4 & 3,2 & 4,8 & 1,5 \\
\hline \multicolumn{12}{|l|}{ MNHN-Na13768 } \\
\hline Mâle 2 & 34,3 & 10,0 & 2,0 & 12,9 & 3,6 & 27,8 & 6,7 & 51,6 & 3,7 & 5,2 & 1,4 \\
\hline Femelle 1 & 27,7 & 11,0 & 1,0 & 9,8 & 2,5 & 25,1 & 5,2 & 53,6 & 2,8 & 4,0 & 1,4 \\
\hline \multicolumn{12}{|l|}{ MNHN-Na13769 } \\
\hline Mâle 3 & 25,2 & 10,0 & 1,0 & 10,2 & 2,7 & 26,5 & 5,4 & 52,7 & 2,4 & 4,1 & 1,7 \\
\hline Femelle 2 & 35,8 & 10,0 & 2,0 & 13,3 & 3,8 & 28,4 & 6,8 & 51,0 & 3,3 & 4,6 & 1,4 \\
\hline Femelle 3 & 31,5 & 10,0 & 2,0 & 11,3 & 2,8 & 24,6 & 6,3 & 56,2 & 2,8 & 3,7 & 1,3 \\
\hline Femelle 4 & 31,1 & 10,0 & 2,0 & 11,8 & 3,5 & 29,7 & 6,5 & 54,7 & 3,2 & 4,7 & 1,4 \\
\hline Femelle 5 & 32,0 & 11,0 & 2,0 & 12,1 & 3,2 & 26,5 & 6,6 & 54,6 & 2,9 & 4,2 & 1,4 \\
\hline
\end{tabular}

Dents $S$ : dents sur la partie supérieure du rostre ; Dents I : den ts sur la partie inférieure du rostre LC : longueur de la carapace ; D1dent : distance entre la $1^{\text {ère }}$ dent et la limite postorbitaire LCD : longueur de la carapace avec dents ; $6 \mathrm{SA}$ : longueur du $6^{\text {ème }}$ segment abdominal 


\begin{tabular}{|c|c|c|c|c|c|c|c|c|}
\hline & Doigt & Paume & Propode & Carpe & Mérus (M) & Ischion (I) & $\% \mathrm{I} / \mathrm{M}$ & Long. totale P2 (mm) \\
\hline \multicolumn{9}{|l|}{ MNHN-Na13767 } \\
\hline Mâle 1 P2L (Holotype) & 8,6 & 9,2 & 17,8 & 9,4 & 6,8 & 3,6 & 52,9 & 37,6 \\
\hline \multicolumn{9}{|l|}{ MNHN-Na13768 } \\
\hline Mâle 2 P2L & 7,6 & 9,6 & 17,1 & 8,1 & 7,6 & 4,4 & 58,1 & 37,3 \\
\hline Femelle $1 \mathrm{P} 2 \mathrm{~L}$ & 3,6 & 3,4 & 7,1 & 3,8 & 4,5 & 4,4 & 99,1 & 19,7 \\
\hline \multicolumn{9}{|l|}{ MNHN-Na13769 } \\
\hline Mâle 3 P2L & 4,7 & 6,5 & 11,2 & 5,6 & 5,1 & 2,8 & 54,7 & 24,6 \\
\hline Femelle 2 P2L & 5,4 & 6,8 & 12,2 & 5,5 & 5,4 & 3,6 & 66,9 & 26,8 \\
\hline Femelle 2 P2C & 4,2 & 4,2 & 8,4 & 4,7 & 4,2 & 3,3 & 77,9 & 20,6 \\
\hline Femelle $3 \mathrm{P} 2 \mathrm{~L}$ & 4,1 & 4,9 & 9,1 & 4,3 & 3,7 & 3,3 & 87,4 & 20,4 \\
\hline Femelle 3 P2C & 3,7 & 3,6 & 7,3 & 3,7 & 3,0 & 2,1 & 70,9 & 16,0 \\
\hline Femelle 4 P2L & 4,4 & 5,3 & 9,6 & 4,6 & 4,4 & 3,5 & 79,5 & 22,2 \\
\hline Femelle 4 P2C & 4,2 & 4,0 & 8,2 & 4,1 & 3,9 & 3,4 & 86,5 & 19,6 \\
\hline Femelle 5 P2L & 3,9 & 3,4 & 7,3 & 3,8 & 3,7 & 3,0 & 83,0 & 17,8 \\
\hline Femelle 5 P2C & 3,9 & 3,4 & 7,3 & 3,8 & 3,7 & 3,0 & 83,0 & 17,8 \\
\hline
\end{tabular}


Chez les 3 dernières paires de péréiopodes des mâles adultes, toutes les jointures ont de nombreuses spinules. Celles-ci sont aussi visibles chez les grandes femelles. En outre, il y a des spinules peu habituelles le long de la marge postérieure du propode et des rangées de poils dans la partie distale du propode de la $5^{\text {ème }}$ patte formant une touffe à son extrémité.

Sur les échantillons mesurés, la taille maximale du corps (céphalothorax plus abdomen) est de 35,8 millimètres.

\section{Coloration}

Le mâle est jaune brun à orange sur le dos et les pinces, blanc à hyalin sur le reste du corps. II possède de petites taches rouges réparties sur le corps, plus denses et organisées en forme de vagues sur le céphalothorax. Les pattes sont plutôt hyalines. Les pinces possèdent de nombreuses épines du doigt jusqu'à l'ischion. Ces épines sont moins développées chez la femelle. La jonction entre chaque article est rougeâtre. La femelle est souvent bleu foncé à noire sur tout le corps. Seule la partie inférieure du céphalothorax et les pattes sont plus claires, jusqu'à tendre vers le vert-bleu. On peut trouver des individus (mâles et femelles) de teinte orangée.

\section{Différenciation}

Macrobrachium feunteuni n. sp. est très proche de Macrobrachium lepidactyloides (De Man, 1892) et de Macrobrachium lepidactylus (Hilgendorf, 1879). Elle diffère de M. lepidactyloides par 9 à 11 dents sur le bord supérieur du rostre versus 10 à 13, par la distance entre la première dent et la limite postérieure de l'orbite représentant 24,6 à $30,7 \%$ de la longueur du céphalothorax versus 39 à $48 \%$, par la longueur de la carapace portant les dents rostrales représentant 51 à $56,2 \%$ de la longueur du céphalothorax versus 38,5 à $45,5 \%$, par les doigts de la plus grande patte de la deuxième paire de péréiopodes des mâles en général plus courts que la paume (versus plus longs) et par plus de dents sur le dactyle. Elle diffère de M. lepidactylus par 6 à 7 dents rostrales placées derrière la limite de l'orbite versus 3 à 5 et par un carpe de la deuxième paire de péréiopodes des mâles toujours plus long que le mérus versus plus court ou égal.

\section{Distribution}

Cette espèce est endémique des îles Marquises. Elle a été trouvée à Nuku Hiva, Hiva Oa, Tahuata et Ua Uka.

\section{Ecologie}

Macrobrachium feunteuni n. sp. fréquente plutôt la partie haute du cours inférieur des rivières et la partie basse du cours moyen. Elle est parfois très abondante. Elle préfère les zones courantes de type radier avec des contre-courants. Elle semble avoir un régime alimentaire de type opportuniste : elle se nourrit de débris organiques et d'algues. Les œufs font un diamètre de 0,45 à $0,6 \mathrm{~mm}$ et sont nombreux et petits.

\section{Derivatio nominis}

L'espèce est nommée en l'honneur de notre collègue Eric Feunteun, ichtyologue à l'université de Rennes, qui a collecté avec nous les échantillons marquisiens lors de la mission « Julien1 » de février 2000. 


\section{DISCUSSION}

Dans les systèmes insulaires de la région Indo-Pacifique, la diversification de la faune et de la flore est fonction de l'éloignement de l'île par rapport aux grands foyers occidentaux de diffusion faunistique et floristique et de l'ancienneté de leur émersion (KEITH et VIGNEUX, 1997). Ultimes groupements d'îles avant les grands vides orientaux, les archipels polynésiens ont filtré un flux d'espèces végétales et animales très amoindri par la sélection de la distance. Maximales dans les îles continentales, la diversité du milieu et la gamme des possibilités qui en découle diminue sensiblement dans les terres récentes nées du volcanisme océanique. Dans les plus grandes et les plus élevées, les oppositions de versants introduisent un facteur de diversité mis à profit par les peuplements naturels. L'isolement et l'ancienneté de certaines îles ont permis le développement d'espèces endémiques.

On constate ainsi des spécificités faunistiques sur chaque archipel. La richesse spécifique est maximale dans l'archipel de la Société (10 espèces dont 6 Macrobrachium) et dans les Australes (10 espèces dont 4 Macrobrachium ainsi qu'une caridine endémique) ; la richesse spécifique est moindre aux Marquises (6 espèces) qui possèdent néanmoins une espèce endémique et aux Gambier ( 6 espèces) ; elle est faible dans les Tuamotu (1 espèce) quasiment dépourvus d'eau superficielle.

Une seule espèce, signalée dans la bibliographie n'a pas été retrouvée. II s'agit d'Atyopsis spinipes que SEURAT (1934) avait signalé aux Gambier. CHACE (1983) avait déjà émis des doutes sur cette affirmation.

L'étude de la richesse spécifique des différents archipels de la Polynésie française, par rapport à d'autres îles de l'Indo-Pacifique, n'a de valeur comparative que dans le cas des îles hautes, bien que le nombre de rivières étudiées soit très différent d'une île à l'autre. La richesse spécifique en crustacés d'eau douce dans les îles polynésiennes est supérieure à celle des îles de la Réunion (9 espèces) (KEITH et al., 1999), mais inférieure à celle de la Nouvelle-Calédonie (33 espèces) (SHORT et MARQUET, 1998).

L'endémisme de la faune d'eau douce aux Marquises est important mais son origine reste problématique selon les connaissances actuelles. En effet, le taux d'endémisme est surprenant pour les îles marquisiennes qui sont moins isolées que d'autres îles de la plaque pacifique et qui présentent pourtant peu ou pas du tout d'endémisme. Les Marquises sont, à côté des îles Hawaï et de l'île de Pâques, celles qui, de toutes les îles ou groupes d'îles en Océanie, présentent le plus haut degré d'endémisme pour les poissons et les mollusques. La description de Macrobrachium feunteuni, endémique des Marquises, est un élément nouveau et complémentaire.

L'explication de ce fort degré d'endémisme donne lieu à de nombreuses interprétations. La plus connue prétend que les ancêtres des espèces endémiques des Marquises devaient être largement distribués dans les différentes îles de la plaque Pacifique. Un évènement vicariant s'est sûrement produit, évènement qui a entraîné l'isolement des Marquises et a permis la divergence des populations. II est évidemment difficile d'identifier la nature de cet évènement. Deux possibilités sont évoquées : à l'intérieur de la plaque Pacifique, l'apparition d'une ancienne fracture entre les Tuamotu et les Marquises, ce qui aurait entraîné l'augmentation de la distance les séparant et l'isolement des Marquises ; ou bien, deuxième possibilité, un courant ancien, responsable de la dispersion des espèces des Tuamotu aux Marquises, a été progressivement modifié et supprimé en même temps que se produisait l'extension linéaire des îles marquisiennes.

Bien que présentant une biodiversité spécifique moindre qu'en d'autres régions tropicales, la Polynésie française est riche de plusieurs espèces endémiques. Le maintien de cette biodiversité originelle ne tient qu'à la préservation de la qualité des milieux 
aquatiques dont certains sont encore relativement préservés comme notamment sur certaines îles des Marquises.

Ce n'est que par une gestion raisonnée des écosystèmes aquatiques fondée sur des connaissances scientifiques de base (biogéographie, écologie, biologie des espèces, dynamique des populations) que l'on pourra éviter la destruction de bon nombre de ces espèces fragiles soumises à la pression grandissante des activités anthropiques.

La structure des associations animales n'explique plus « l'histoire natrurelle » des milieux et de leurs peuplements associés mais traduit également les conflits d'usages liés aux activités humaines (modification des biotopes, introduction d'espèces exogènes compétitrices ou vectrices de pathologies nouvelles.

II convient dans ce contexte de ne pas oublier la valeur de bioindicateur de qualité des milieux des espèces autochtones, et de garder à l'esprit que dans ces milieux dulçaquicoles du Pacifique encore peu étudiés, certaines pourraient avoir déjà disparu avant même d'avoir été décrites.

\section{BIBLIOGRAPHIE}

ADAMSON A.M., 1935. Non marine invertebrate fauna of the Marquesas (exclusive of insects). Occasional Paper Bernice P. Bishop Museum., 11 (10), 1-39.

ATKINSON J.M., 1977. Larval development of a freshwater prawn, Macrobrachium lar (Decapoda, Palaemonidae), reared in the laboratory. Crustaceana, 33 (2), 119-132.

BOUVIER E.L., 1925. Recherches sur la morphologie, les variations, la distribution géographique des crevettes de la famille des Atyidés. Encyclopédie Entomologique, Paris, 4, 1-370.

CHACE F.A. Jr, 1983. The Atya-like shrimps of the Indo-Pacific Region (Decapoda: Atyidae). Smithsonian contributions to zoology, 384, 1-54.

EDMONDSON C.H., 1935. Atyidae of southern Polynesia. Occasional Paper Bernice P. Bishop Museum., 11 (3), 1-19.

EDMONDSON C.H., 1951. Some central Pacific crustacean. Occasional Paper Bernice $P$. Bishop Museum., 20 (13), 183-243.

EGE V., 1939. A revision of the genus Anguilla Shaw: a systematic, phylogenetic and geographical study. The Carlsberg foundation's oceanographical expedition round the world 1928-30 and previous « Dana »-expeditions. Dana Report, 16 (3), 1-256.

FOSSATI O., MOSSERON M., KEITH P., 2002. Atyidae (Crustacea Decapoda) macro-and micro- distribution in the rivers of Nuku-Hiva island (French Polynesia). Hydrobiologia (sous presse).

FOURMANOIR P., GRIESSINGER J.M., PLESSIS Y., 1974. Faune ichtyologique des Gambier. Cahier du Pacifique, 18 (2), 543-559.

FOWLER H.W., 1932. Freshwater fishes from the Marquesas and Society islands. Occasional Paper Bernice P. Bishop Museum., 9 (25), 1-11.

GOSSET C., LAMARQUE P., CHARLON N., 1971. Un nouvel appareil de pêche électrique portable : le « Martin-pêcheur ». Bull. Fr.. Piscic., 242, 33-46.

GRAND S., 1972. Contribution à l'étude préliminaire sur la biologie, la systématique et l'écologie des Macrobrachium (Palaemonidae) de Tahiti. Thèse, Université de Montpellier, $130 \mathrm{p}$.

HERRE A.W., 1931. A list of fishes collected at Moorea, one of the Society islands, being the first record from this island. J. Pan. Pacif. Res. Inst., 6 (4), 10 p.

HERRE A.W., 1932. A check list of fishes recorded from Tahiti. J. Pan. Pacif. Res. Inst., 7 (1), 2-6.

HOBBS H.H. Jr, HART C.W. Jr, 1982. The shrimps genus Atya (Decapoda: Atyidae). Smithsonian contributions to zoology, 364, 1-143. 
HOLTHUIS L.B., 1950. The decapoda of the Siboga expedition. The Palaemonidae collected by the Siboga and Snellius expeditions with remarks on other species. I. Subfamily Palaemonidae. Siboga Expeditie, Leiden, 39, 1-268.

HOLTHUIS L.B., 1952. On some Indo-Westpacific Palaemoninae (Crustacea decapoda Caridea). Zoologishe mededelingen, 31 (18), 201-211.

HOLTHUIS L.B., 1980. Shrimps and prawns of the world, an annnoted catalogue of species of interest to fisheries. FAO Fisheries Synopsis, 125 (1), 1-230.

KEITH P., VIGNEUX E. 1997. Inventaire des poissons et crustacés d'eau douce de Polynésie française. Rap. MNHN, EPHE, CSP, Del. Env. Polynésie, 110 p.

KEITH P., VIGNEUX E., BOSC P., 1999. Atlas des poissons et crustacés d'eau douce de la Réunion. Patrimoines naturels (M.N.H.N./SPN), 39, 136 p.

KENDALL W.C., GOLDSBOROUGH E.L. 1911. Reports on the scientifics results of the expedition to the tropical pacific. The shore fishes. Mus. Comp. Zool. Mem., 26 (7), 341-344.

MARQUET G., 1991. Freshwater crustaceans of French Polynesia: taxonomy, distribution and biomass (Decapoda). Crustaceana, 61 (2), 126-140.

MARQUET G., GALZIN R., 1992. Systématique, répartition et biomasse des poissons d'eau douce de Polynésie française. Cybium, 16 (3), 245-259.

PLESSIS Y., MAUGE L.A., 1978. Ichtyologie des îles Marquises. Cah. Pacif., 21, 215-235.

PLESSIS Y., 1973. Etude préliminaire de la faune ichtyologique de Moorea. Cah. Pacif., 17, $79-88$.

PLESSIS Y., 1980. Etude ichtyologique de Tubuaï, archipel des Australes (Polynésie). Cah. Indo-Pacif., 2 (3), 255-269.

PLESSIS Y., 1987. Etude ichtyologique de Rapa. DIRCEN SMCB, LEFRANCQ et Cie, Eds., Candé, 215-230.

POLL M., 1942. Les poissons de Tahiti recueillis par G.A. De Witte. Bull. Mus. Roy. Hist. Nat. Belgique, 18 (61), $20 \mathrm{p}$.

RANDALL J.E., 1973a. Tahitian fish names and preliminary checklist of the fishes of the Society Islands. Occasional Paper Bernice P. Bishop Museum., 24 (11), 167-203.

RANDALL J.E., 1973b. Rapan fish names and a preliminary checklist of the fishes of the Society islands. Occasional Paper Bernice P. Bishop Museum., 24 (15), 291-306.

RANDALL J.E., 1978. Marine Biological and Archeological Expedition to Southeast Oceania. Nat. Geogr. Soc. Res. Rep., 1969 Projects, 473-495.

RANDALL J.E., 1985. Fishes. In : Fauna and Flora, a first compendium of French Polynesian sea-dwellers. Proc. Fifth Int. Coral Reff Cong., Tahiti, 1, 462-481.

RANDALL J.E., SMITH C.L., FEINBERG M.N., 1990. Report on fish collections from Rapa, French Polynesia, Amer. Mus. Novit., 2966, 44 p.

SEURAT L.G., 1934. La faune et le peuplement de la Polynésie française. Mém. Soc. Biogéogr., 4, 41-74.

SHORT J., MARQUET G., 1998. New records of freshwater Palaemonidae from New Caledonia. Zoosystema, 20 (2), 401-410. 


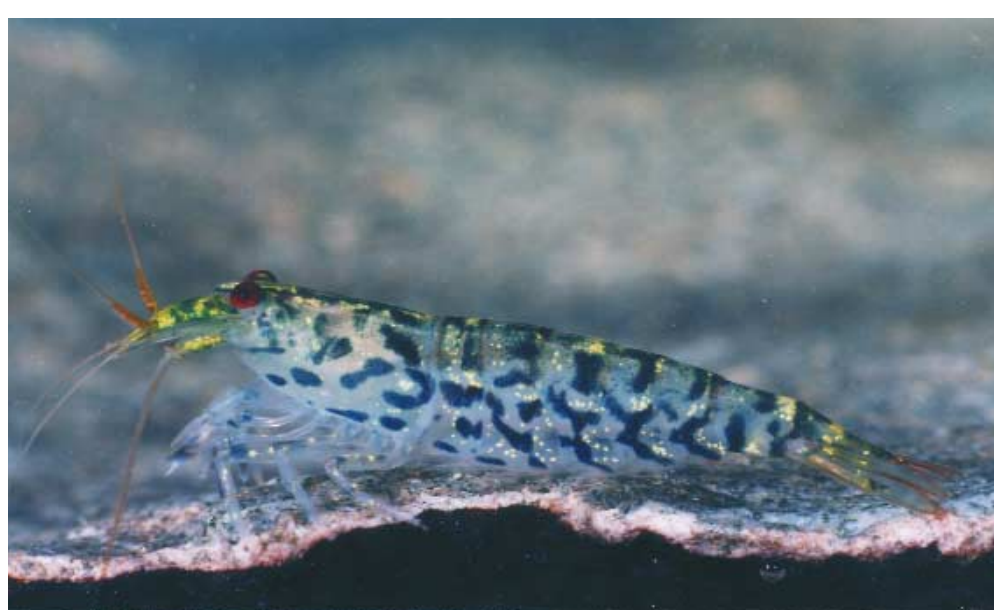

Photo 1 : Atyoida pilipes.

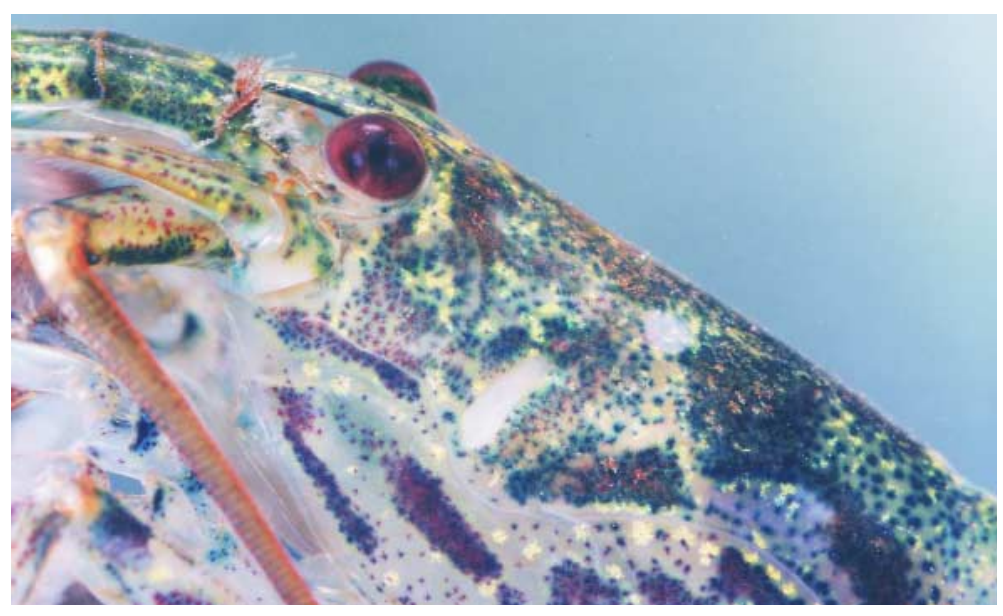

Photo 2 : Atyoida pilipes.

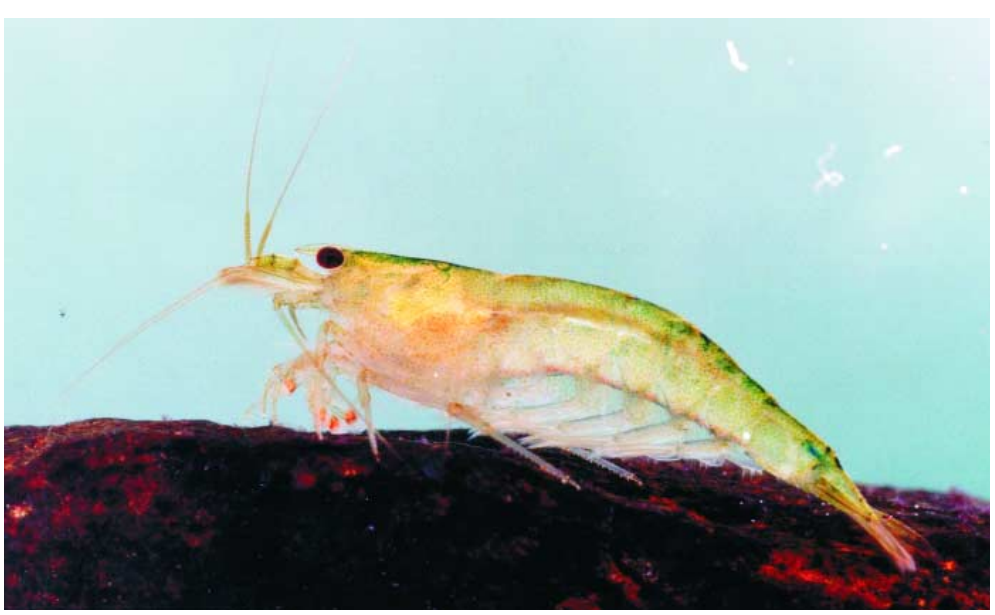

Photo 3 : Caridina rapaensis.

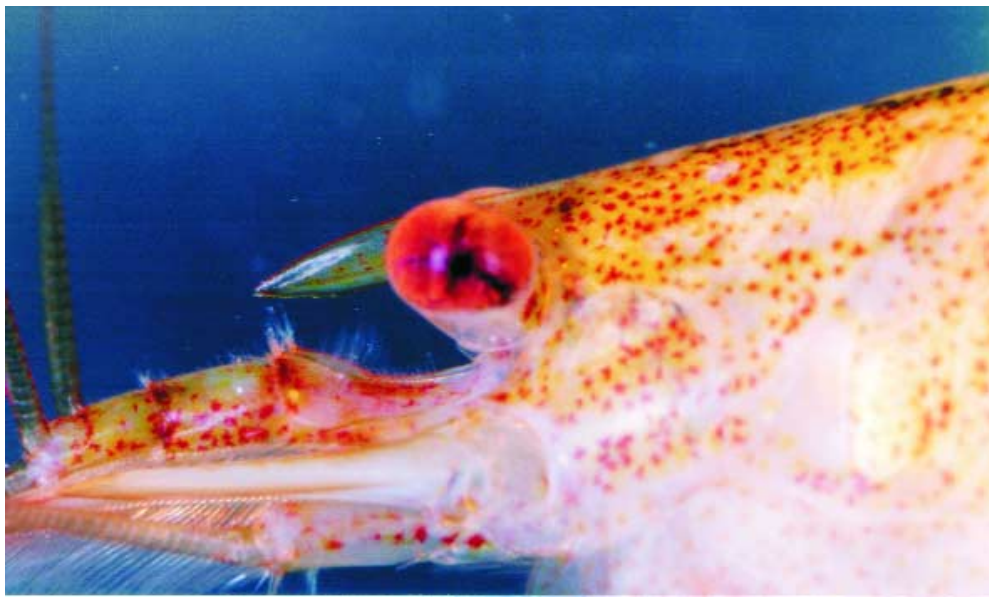

Photo 4 : Caridina rapaensis. 


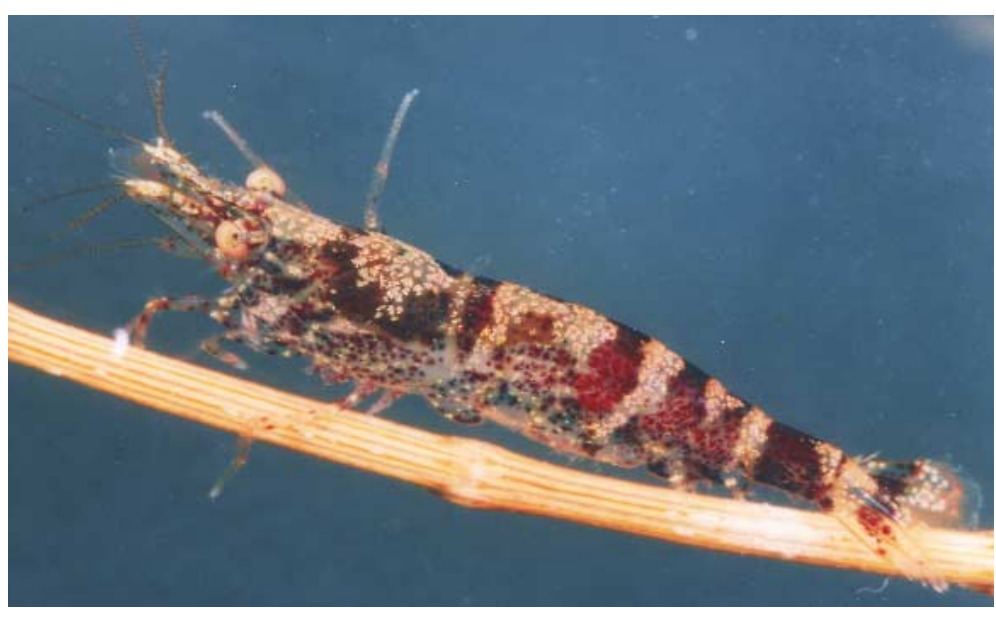

Photo 5 : Caridina serratirostris.

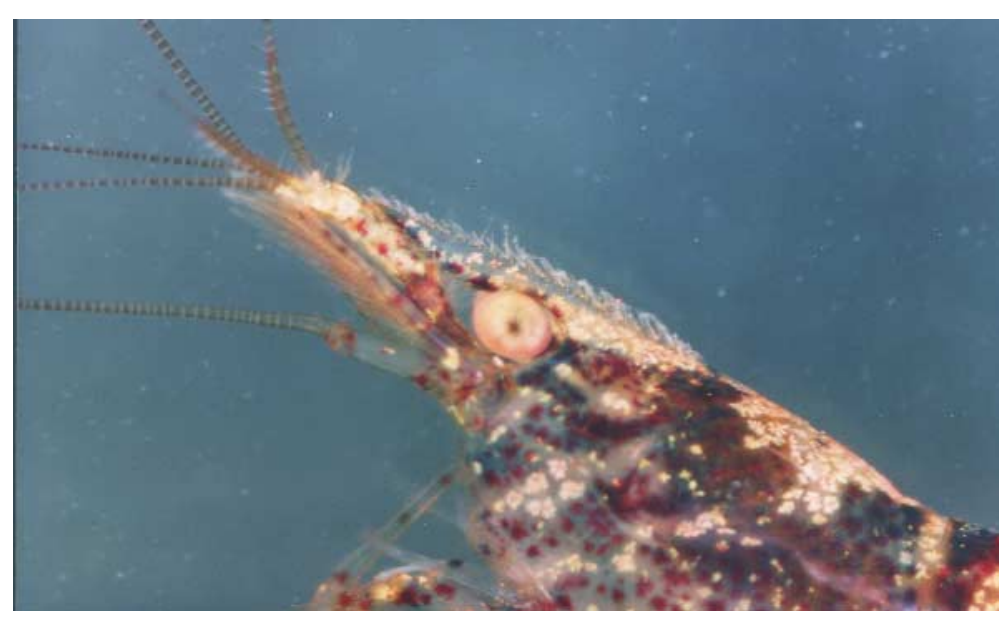

Photo 6 : Caridina serratirostris.

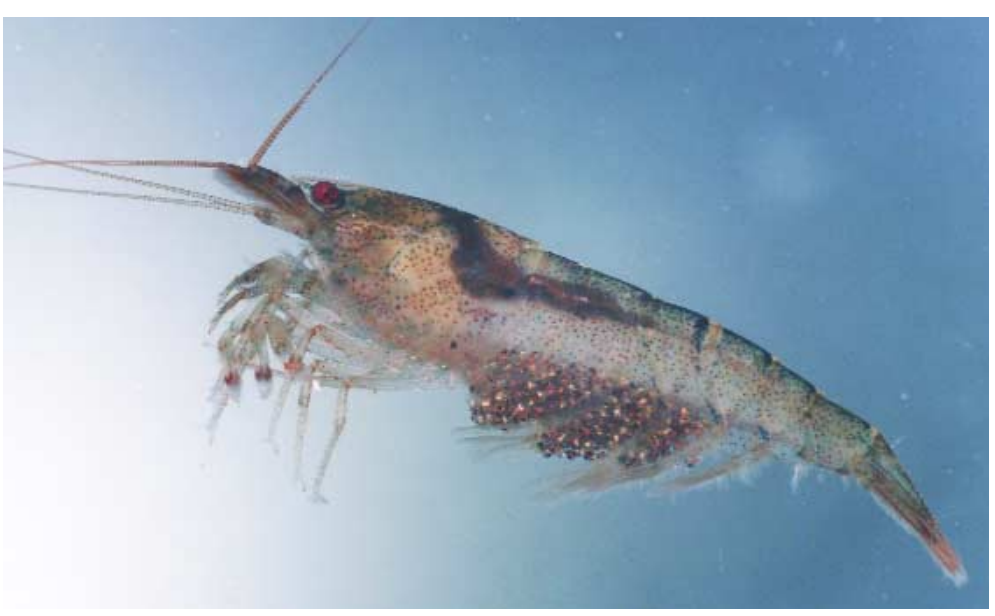

$\stackrel{\infty}{s}$

7

$\stackrel{8}{8}$

w

$\stackrel{\vec{U}}{\overrightarrow{\vec{\Delta}}}$

Photo 7 : Caridina weberi.

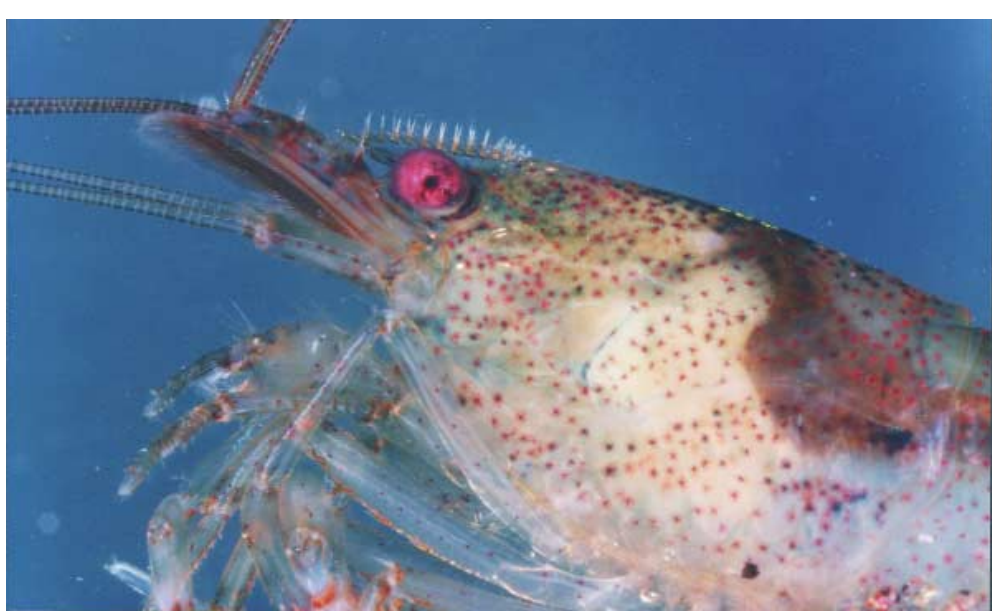




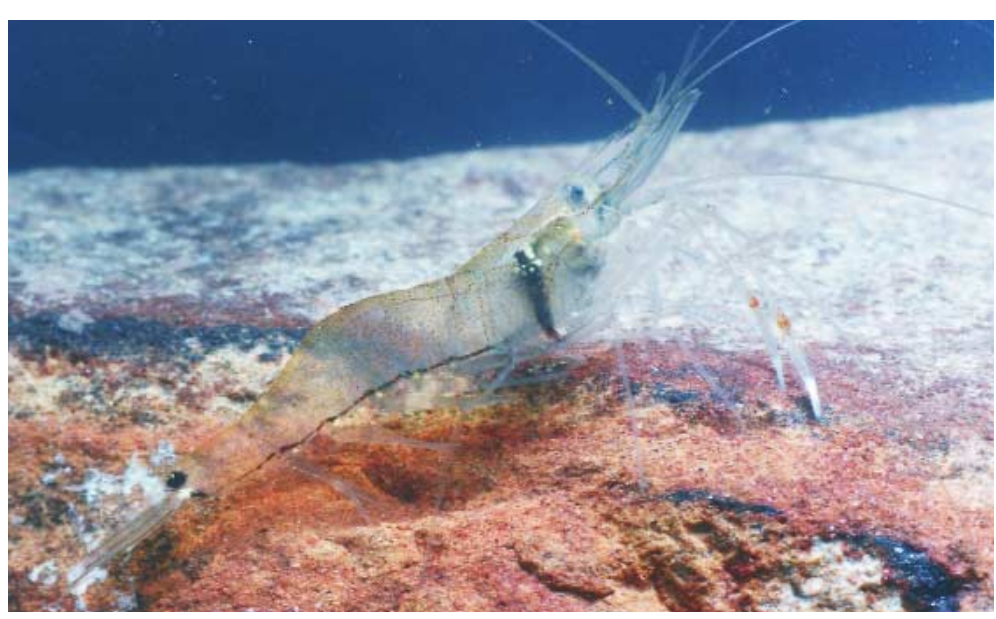

Photo 9 : Palaemon concinnus.

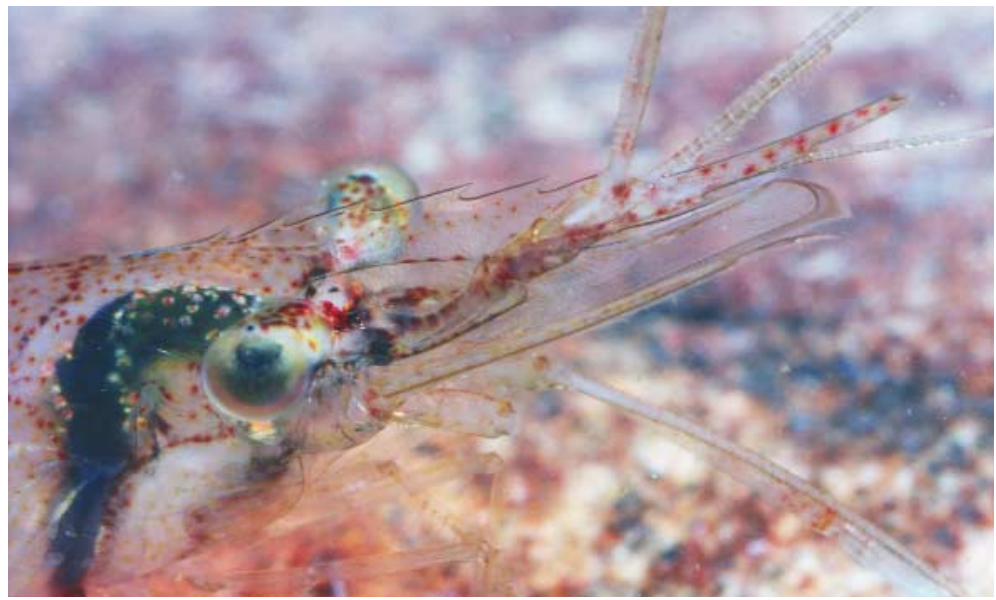

Photo 10 : Palaemon concinnus.

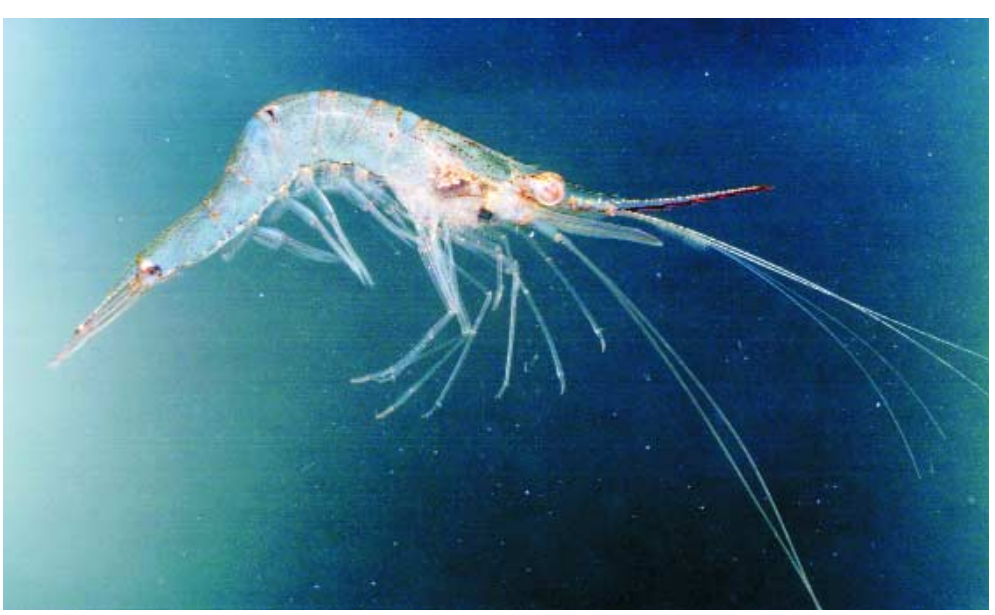

Photo 11 : Palaemon debilis.

$\frac{1}{\partial}$

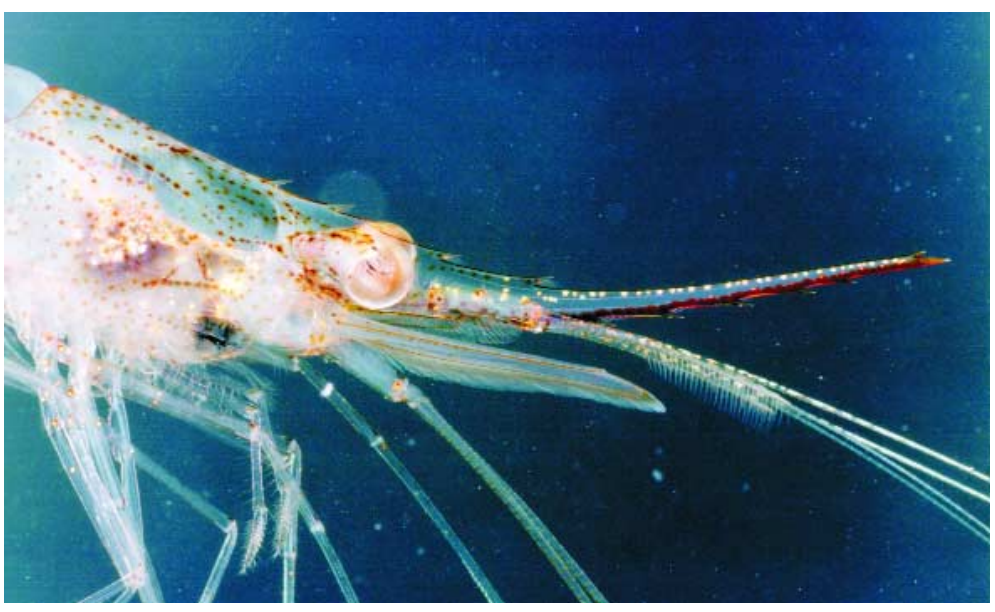

Photo 12 : Palaemon debilis. 


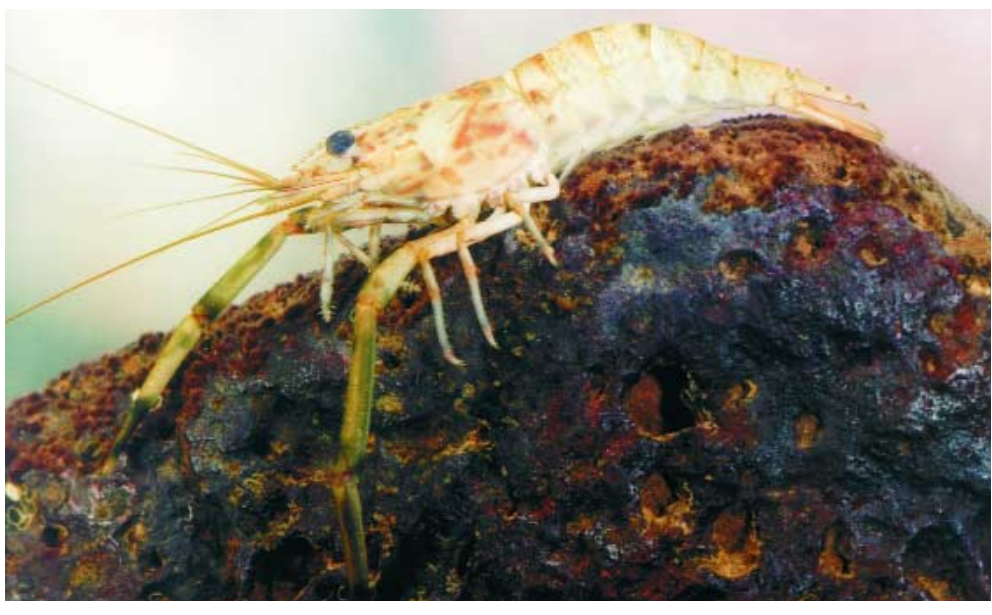

Photo 13 : Macrobrachium aemulum.

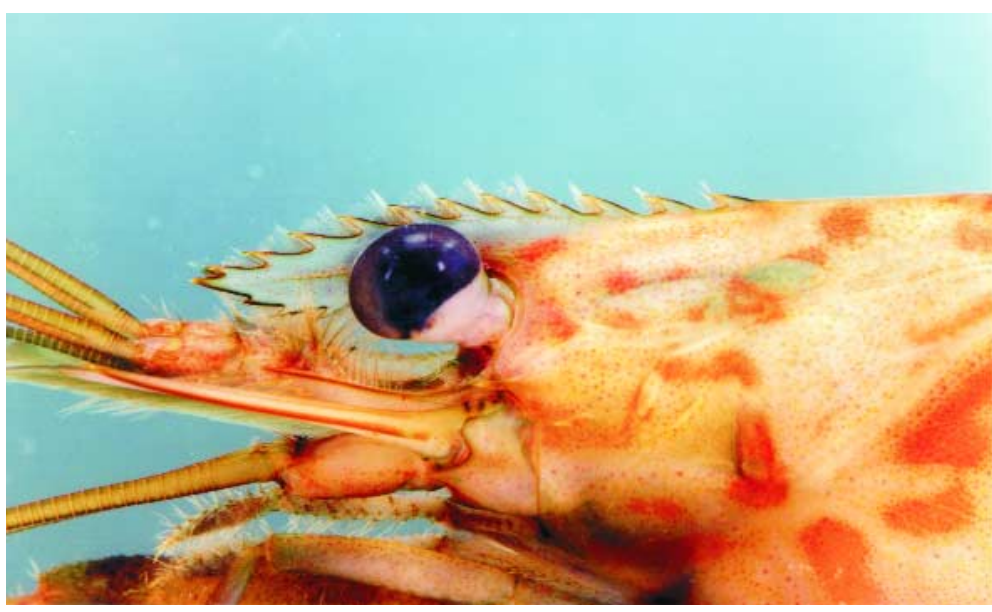

Photo 14 : Macrobrachium aemulum.

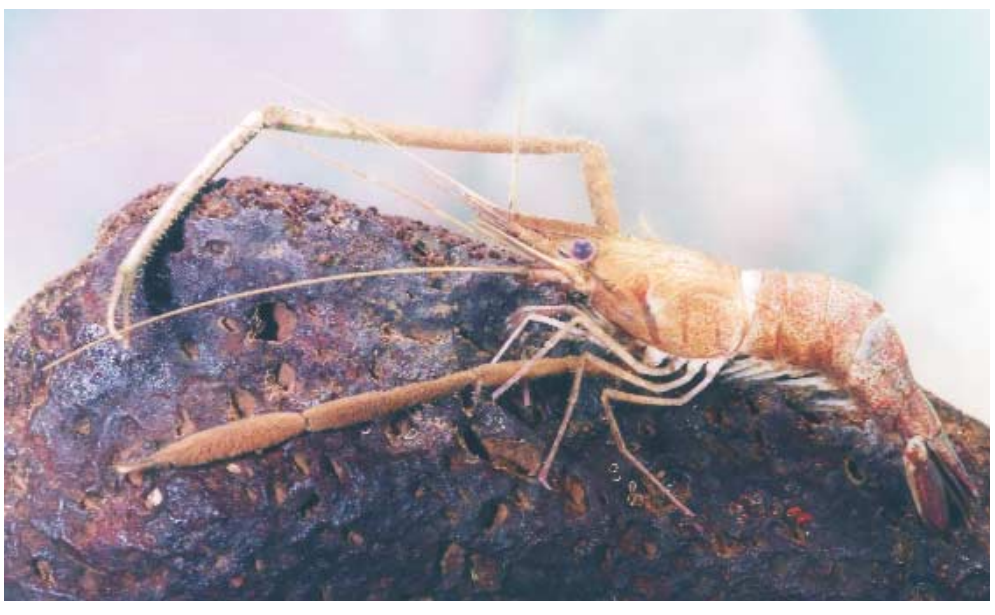

Photo 15 : Macrobrachium australe.

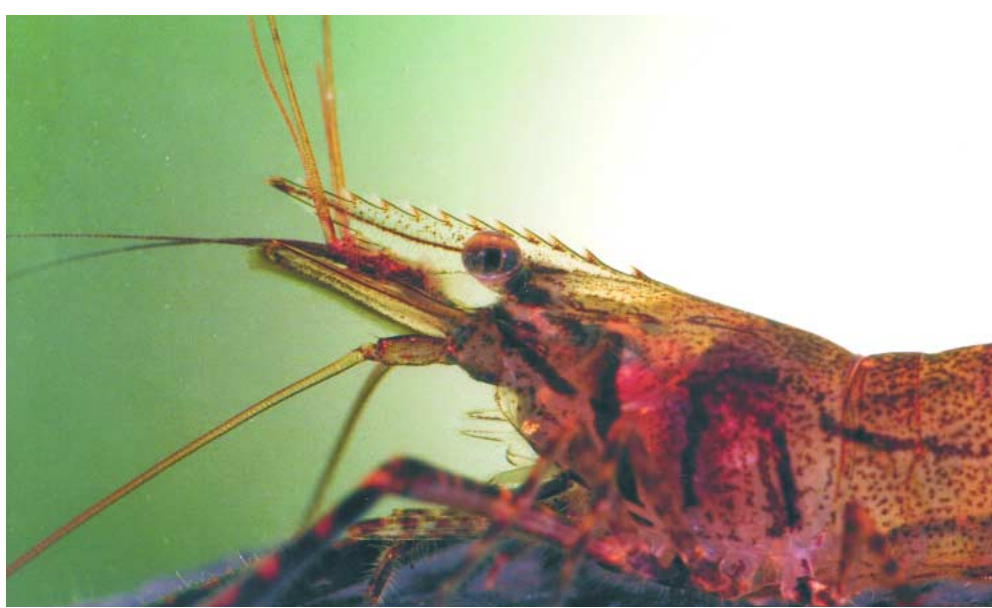

Photo 16 : Macrobrachium australe. 


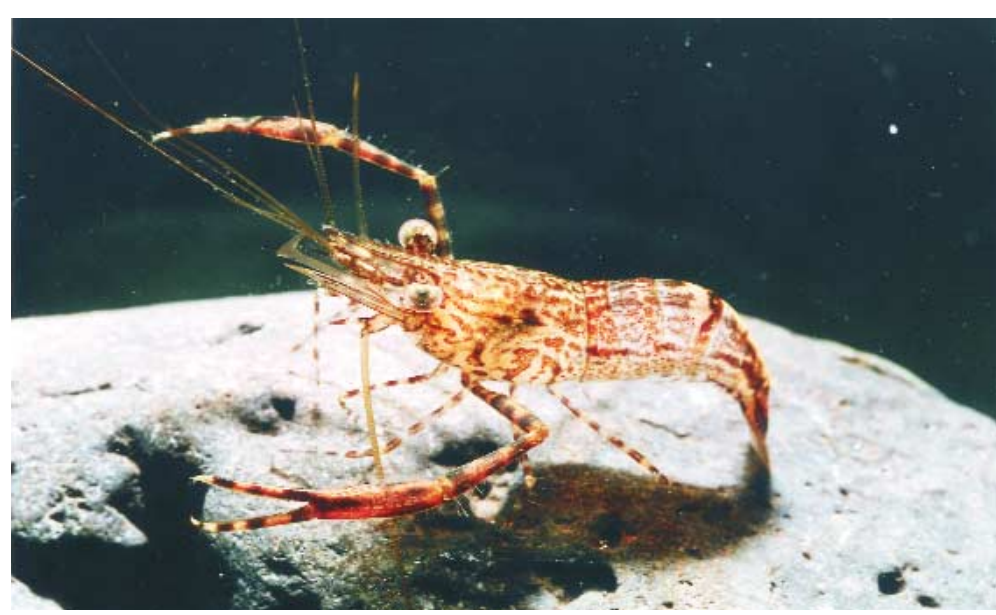

Photo 17 : Macrobrachium grandimanus.

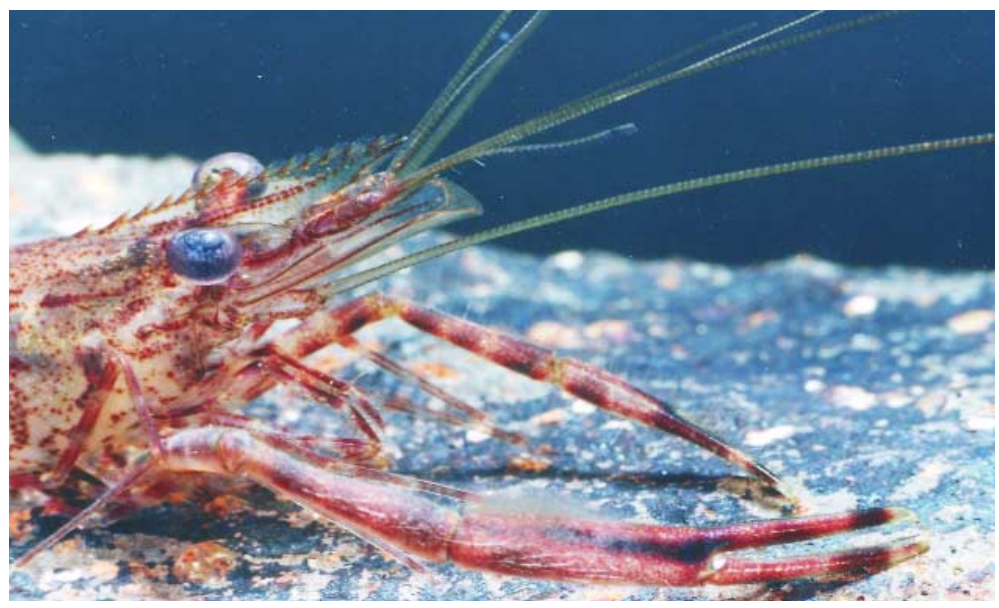

Photo 18 : Macrobrachium grandimanus.

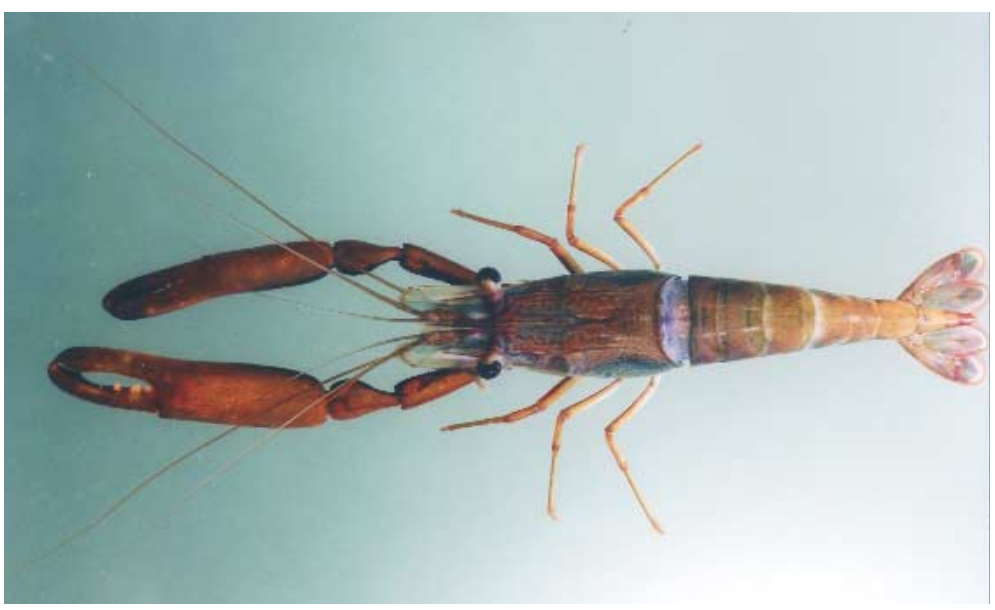

Photo 19 : Macrobrachium latimanus.

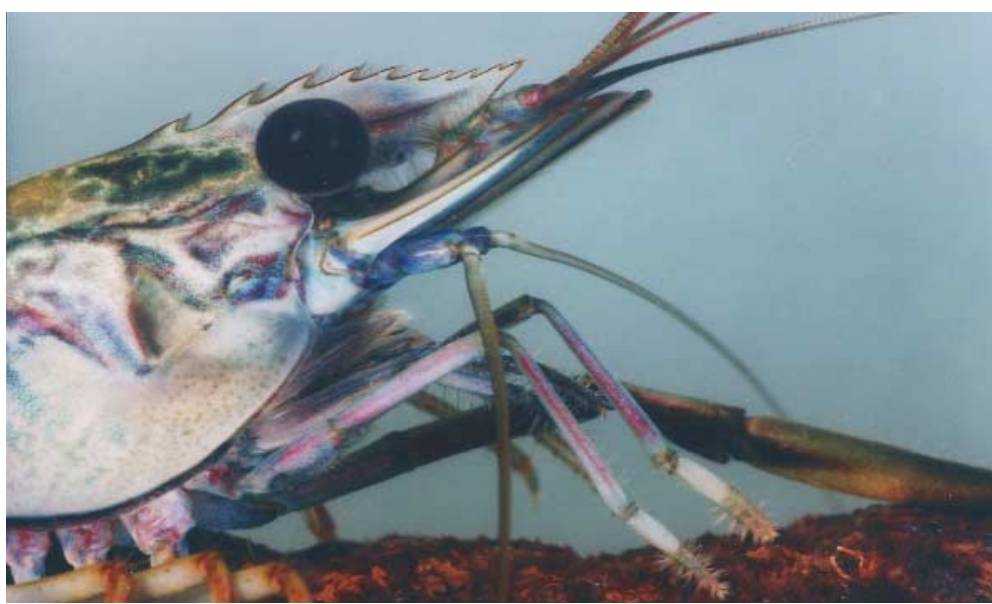

Photo 20 : Macrobrachium latimanus. 


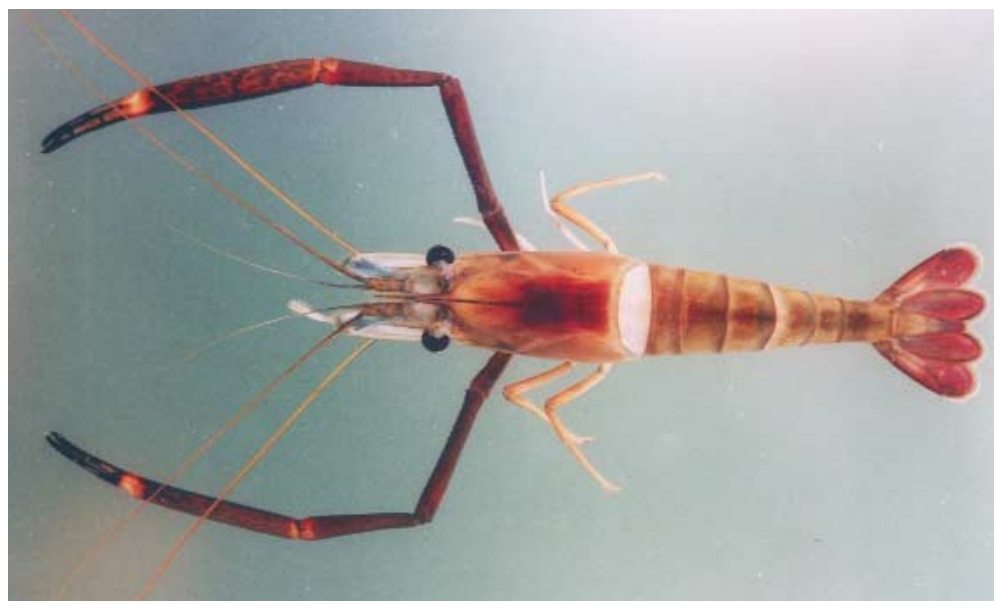

Photo 21 : Macrobrachium lar.

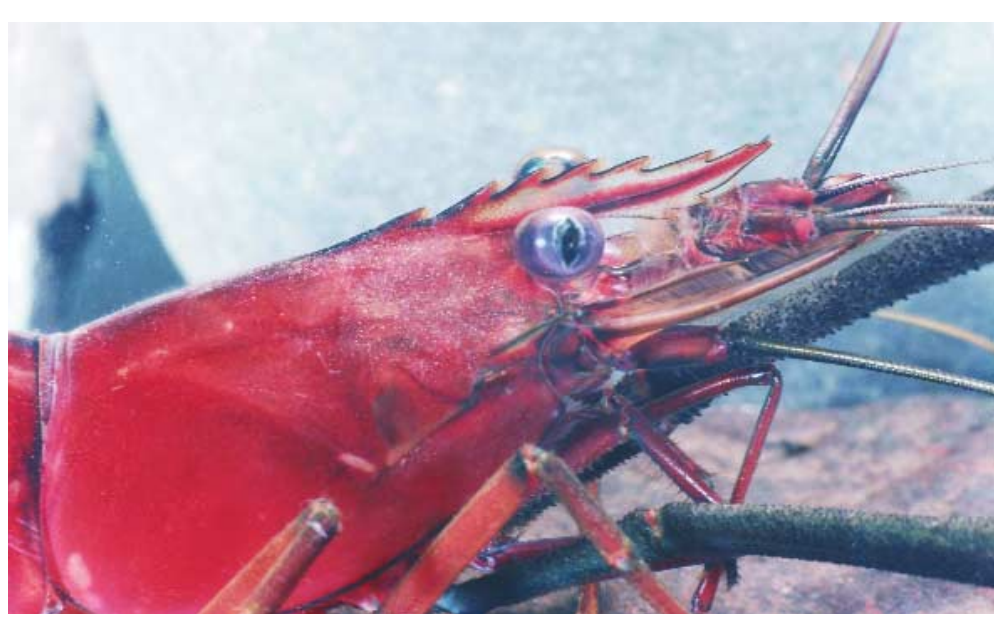

Photo 22 : Macrobrachium lar.

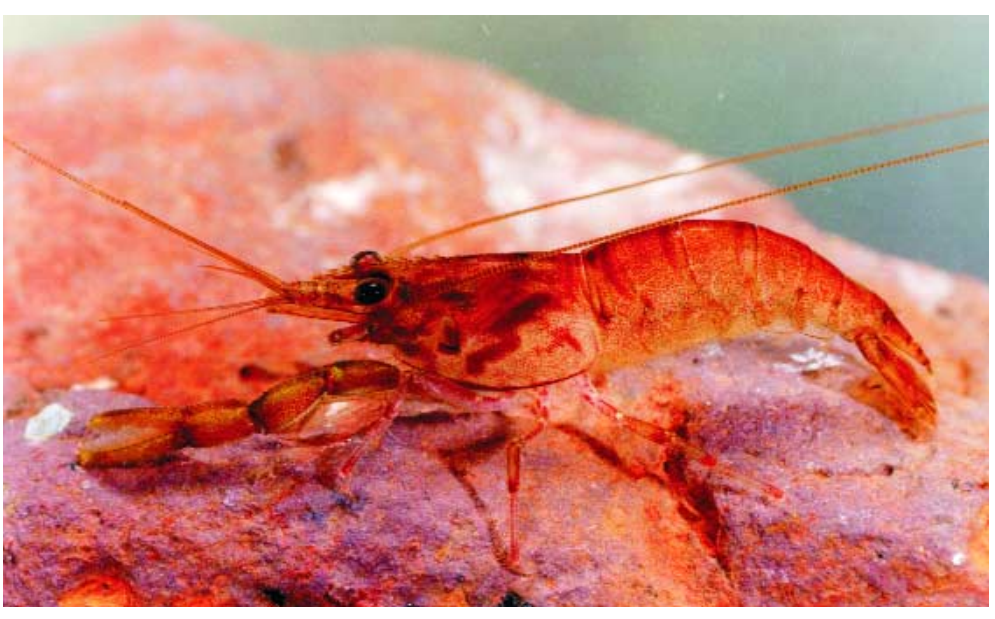

Photo 23 : Macrobrachium lepidactyloides.

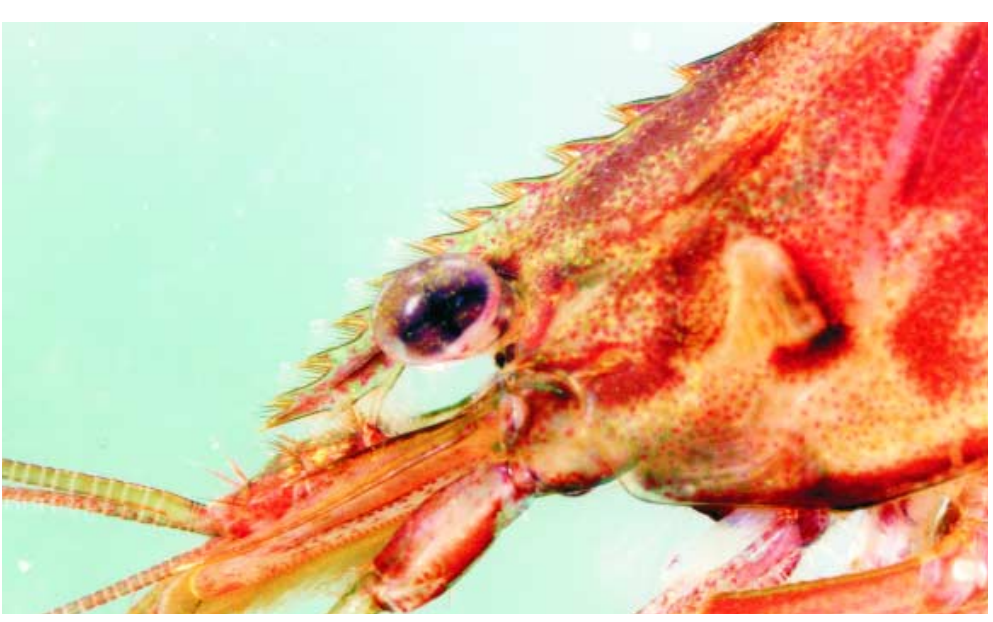

Photo 24 : Macrobrachium lepidactyloides. 


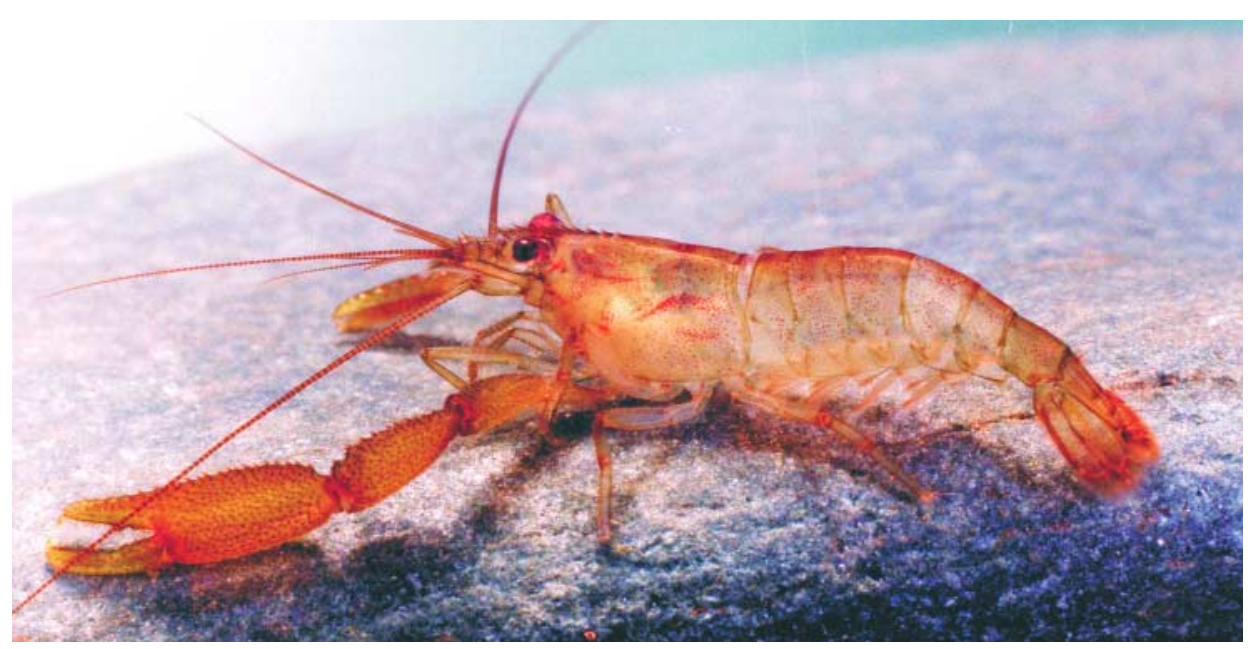

Photo 25 : Macrobrachium feunteuni n. sp. (mâle).

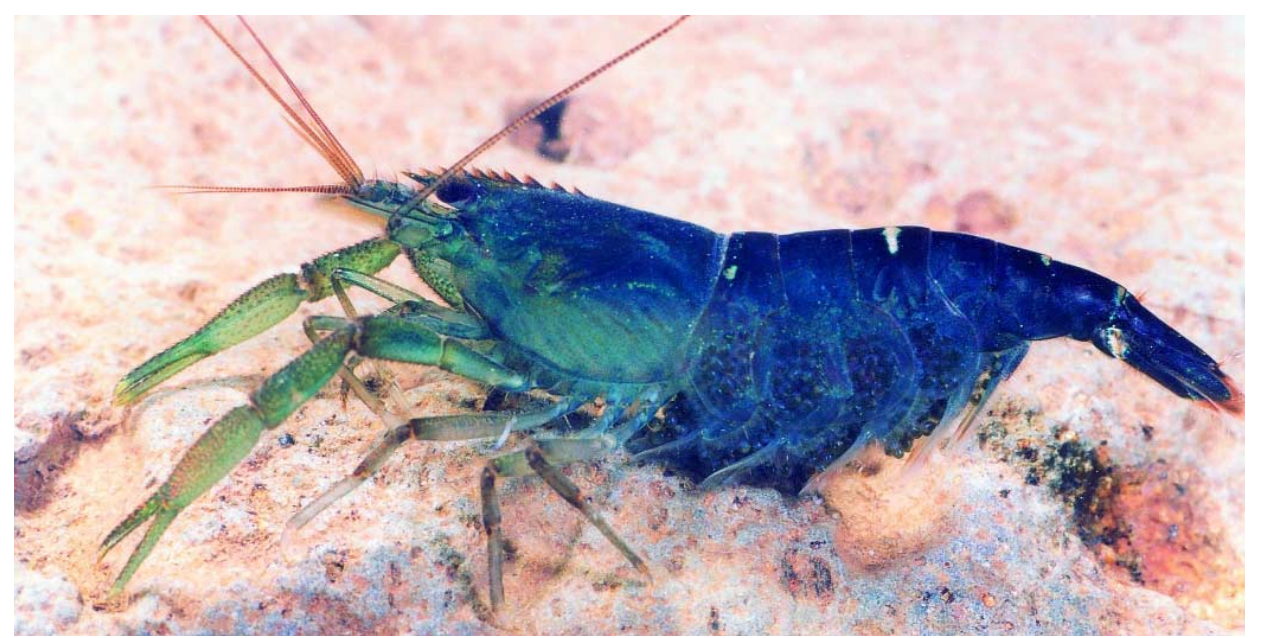

Photo 26 : Macrobrachium feunteuni n. sp. (femelle).

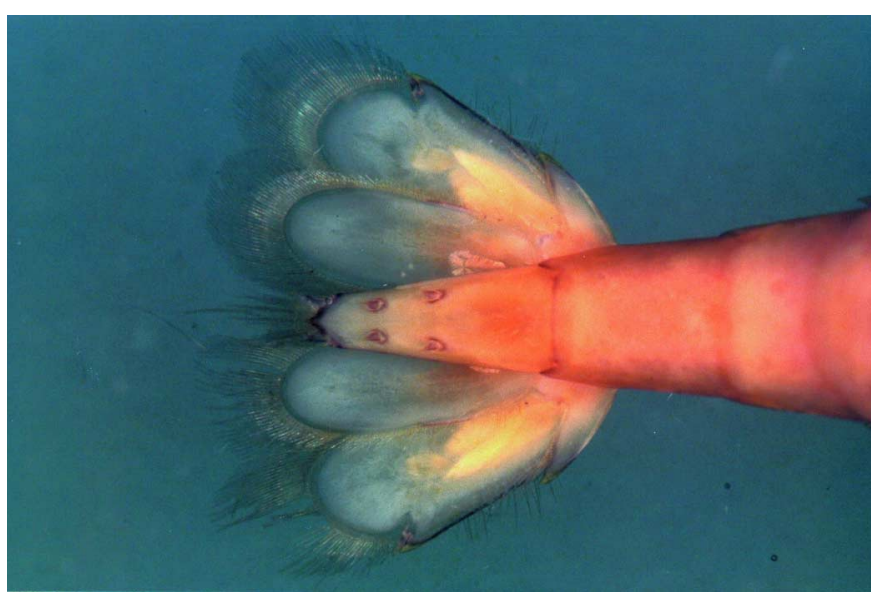

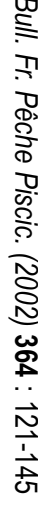

Photo 27 : Macrobrachium feunteuni n. sp.

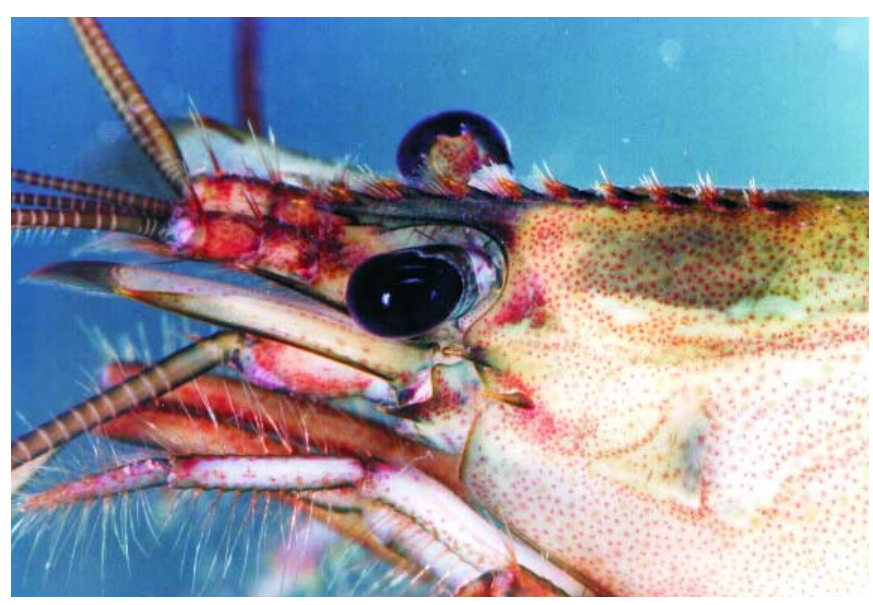

Photo 28 : Macrobrachium feunteuni n. sp. 


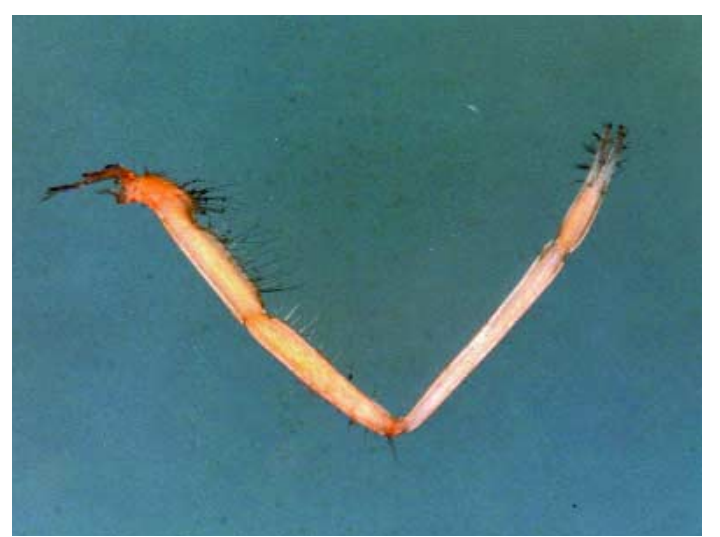

Photo 29 : Macrobrachium feunteuni n. sp. Péréiopode 1 (P1)

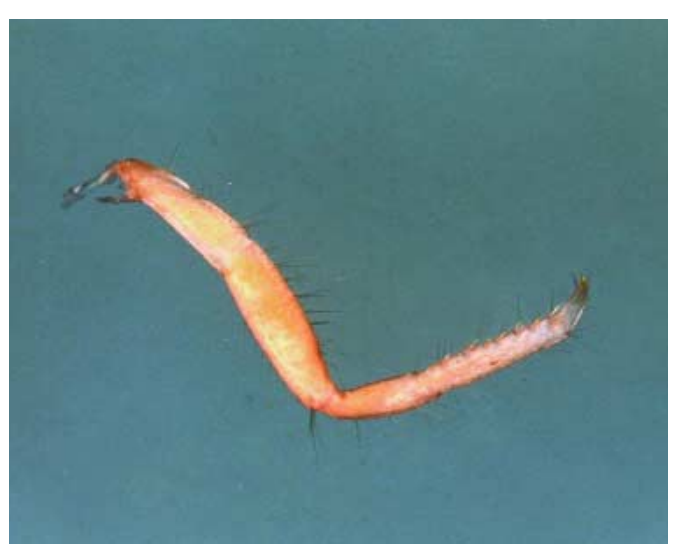

Photo 30 : Macrobrachium feunteuni n. sp. Péréiopode 3 (P3)

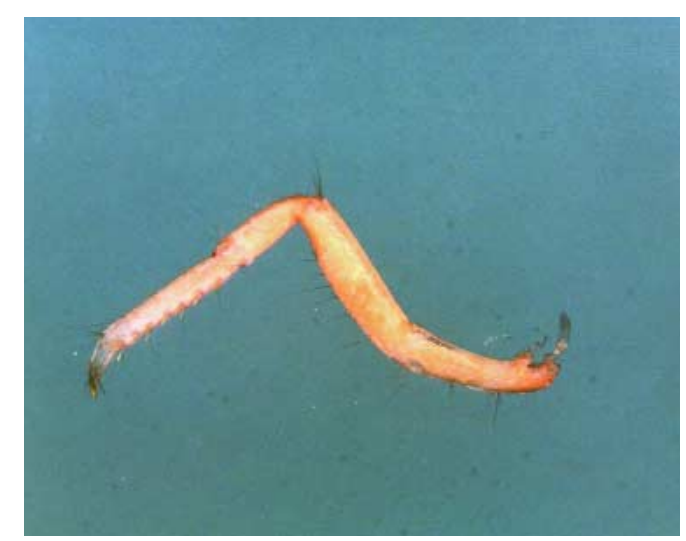

Photo 31 : Macrobrachium feunteuni n. sp. Péréiopode 4 (P4).

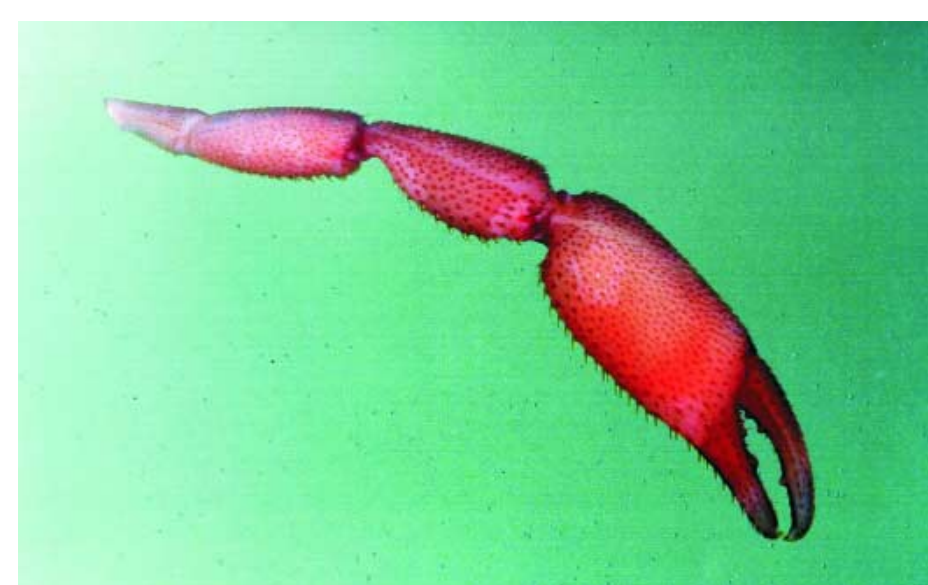

Photo 32 : Macrobrachium feunteuni n. sp. (mâle). Péréiopode 2 (P2).

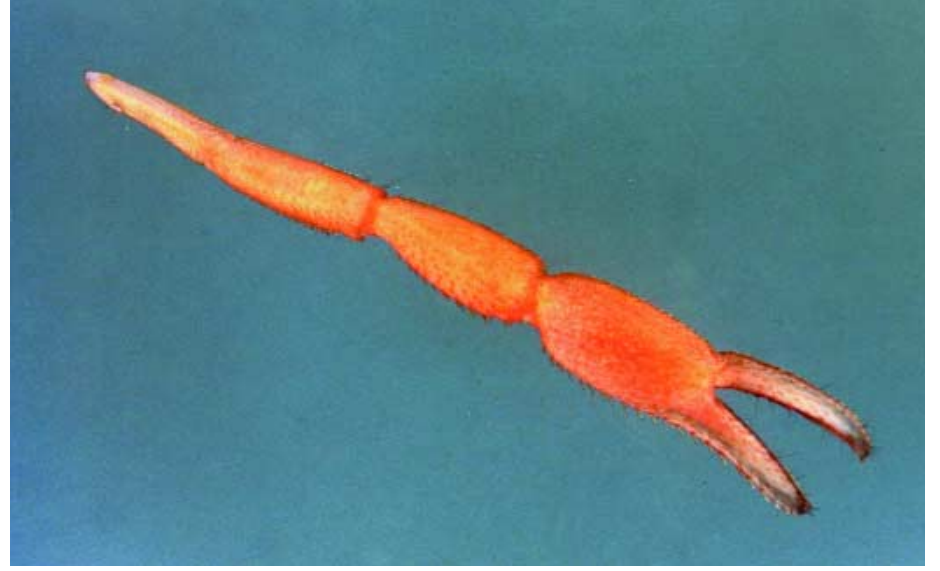

Photo 33 : Macrobrachium feunteuni n. sp. (femelle). Péréiopode 2 (P2) 
